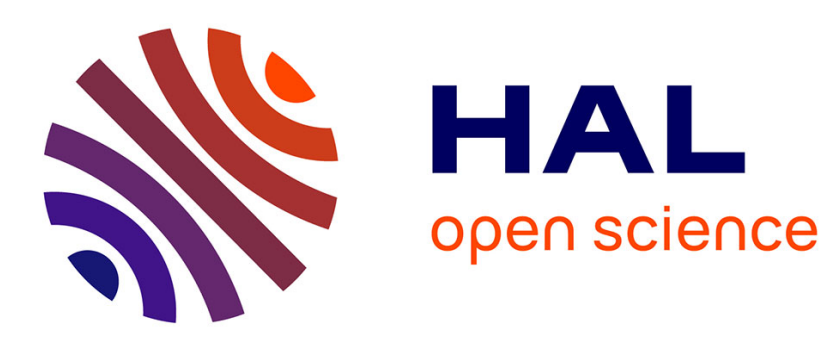

\title{
Computing multiple periodic solutions of nonlinear vibration problems using the harmonic balance method and Groebner bases
}

Aurelien Grolet, Fabrice Thouverez

\section{- To cite this version:}

Aurelien Grolet, Fabrice Thouverez. Computing multiple periodic solutions of nonlinear vibration problems using the harmonic balance method and Groebner bases. Mechanical Systems and Signal Processing, 2015, 52-53, pp.529-547. 10.1016/j.ymssp.2014.07.015 . hal-02121532

\section{HAL Id: hal-02121532 \\ https://hal.science/hal-02121532}

Submitted on 18 Dec 2019

HAL is a multi-disciplinary open access archive for the deposit and dissemination of scientific research documents, whether they are published or not. The documents may come from teaching and research institutions in France or abroad, or from public or private research centers.
L'archive ouverte pluridisciplinaire HAL, est destinée au dépôt et à la diffusion de documents scientifiques de niveau recherche, publiés ou non, émanant des établissements d'enseignement et de recherche français ou étrangers, des laboratoires publics ou privés. 


\title{
Computing multiple periodic solutions of nonlinear vibration problems using the harmonic balance method and Groebner bases
}

\author{
Aurelien Grolet*, Fabrice Thouverez \\ Ecole Centrale de Lyon, Laboratoire de Tribologie et Dynamique des Systèmes, 36 avenue Guy de Collongue, 69134 Ecully Cedex, France
}

This paper is devoted to the study of vibration of mechanical systems with geometric nonlinearities. The harmonic balance method is used to derive systems of polynomial equations whose solutions give the frequency component of the possible steady states. Groebner basis methods are used for computing all solutions of polynomial systems. This approach allows to reduce the complete system to an unique polynomial equation in one variable driving all solutions of the problem. In addition, in order to decrease the number of variables, we propose to first work on the undamped system, and recover solution of the damped system using a continuation on the damping parameter. The search for multiple solutions is illustrated on a simple system, where the influence of the retained number of harmonic is studied. Finally, the procedure is applied on a simple cyclic system and we give a representation of the multiple states versus frequency.

\section{Introduction}

The present work is devoted to studying nonlinear dynamic systems subjected to polynomial nonlinearities. It is well known that nonlinear systems may exhibit complex dynamics and, in particular, multiple steady-state solutions [1-3]. Some nonlinear systems [1] can even have a countable infinity of periodic solutions, which makes the search for all solutions very difficult, if not impossible. The goal of this paper is to propose a method based on the harmonic balance method (HBM), Groebner bases and continuation methods that allow deriving multiple solutions of a nonlinear dynamic system (free or forced). As the HBM introduce an approximation (truncation in the number of retained harmonics), only a finite number of solution can be obtained, and here, "multiple solutions" are used in the sense "as many solutions as possible relative to the HBM approximation", or in other words "all solutions of the HBM equations".

The harmonic balance method (HBM) is widely used in finding approximation to periodic solutions of nonlinear differential equations; the main HBM step consists of transforming the set of nonlinear differential equations into a set of nonlinear algebraic equations, which in turn can be solved to yield the Fourier coefficients of a particular solution. The HBM is a very efficient method and is capable of handling nearly all types of nonlinearities (polynomial [3], friction [4], contact [5]) regardless of their amplitudes (strong or weak nonlinearities). The Newton-Raphson algorithm and continuation methods are typically applied to the algebraic equations in order to track the solution as frequency varies [6] (note: by

\footnotetext{
* Principal corresponding author.

E-mail addresses: aurelien.grolet@ec-lyon.fr (A. Grolet), fabrice.thouverez@ec-lyon.fr (F. Thouverez).
} 
frequency we meant excitation frequency (in the forced case), and natural frequency (in the unforced case)). With a change in frequency, bifurcations may occur and several new branches of solutions can be computed from these bifurcation points depending on the specific bifurcation type [6,3]. The locations of bifurcation points and tangent directions can be computed using Newton-Raphson algorithms in a unique algebraic system (e.g. see [6]). Determining all bifurcation points however is not a simple task as some points may be easily overlooked (e.g. when the continuation step length is too high or when the determinant of the Jacobian matrix tends to zero but does not change sign). Moreover, if some branches of solutions are disconnected (in the sense that they do not arise from bifurcation points of previous branches), then the continuation algorithms would fail to detect them and new tools would be needed to solve the algebraic system of equations induced by the HBM.

Several methods for finding multiple solutions of dynamic or algebraic systems have been proposed in the literature [3,6-16]; their reviews and presentations will be provided in Section 3. We will specifically focus on the use of Groebner bases for solving the polynomial system of equations generated from the HBM. A Groebner basis can be viewed as a rewriting of the original polynomial system to be solved, yet with additional properties (e.g. in some cases in triangular form) that facilitate the resolution step. The use of a Groebner basis in the field of structural mechanics has already been proposed in a number of studies, including [17,18]. In [17], Groebner bases are used to determine multiple static equilibria of geometrically nonlinear plates subjected to static forces. Before finding solutions, the structural system is reduced using a few modal shapes and the Ritz method, thus leading to a polynomial system with a few degrees of freedom ( 5 max.) describing the static solution of the structural system. The author then computes a (triangular) Groebner basis of this reduced equation and solves the resulting (triangular) system by means of lifting. In [18], Groebner bases are used to compute the solution of nonlinear free vibration of geometrically nonlinear composite plates. The author also reduces the system using the Ritz method and moreover assumes a harmonic response of the structure, thereby transforming the nonlinear differential equations into a set of polynomial algebraic equations (equivalent to an HBM with 1 harmonic). Once again, Groebner bases are computed in triangular form and the system is solved by deriving an expression of the nonlinear frequency vs. motion amplitude. In both studies, the author notes that their reduced model has been limited in size due to the significant increase in Groebner basis computation time with the number of variables (e.g. see [17]: “..., it creates the need for 11 unknown constants and this cannot be accomplished with the computer available for the current studies.").

In this paper, we propose using Groebner bases [11] to solve the set of algebraic equations given by the HBM in order to find multiple steady-state solutions. Unlike previous studies, this one will allow us to identify dynamic solutions of the structural system in the forced case. In order to reduce the number of variables (and therefore the computation time) in the search for multiple solutions, we will first search for solutions of the undamped problem, and then use a damping continuation procedure to recover solutions of the damped system.

The paper will be organized as follows: Section 2 will describe the application of the harmonic balance method along with the continuation methods. Section 3 will then focus on solving the polynomial system and display the resolution method used in this paper. Lastly, Section 4 will present a numerical application on a simple example corresponding to an 8-dof cyclic structure with cubic nonlinearities. The set of all steady-state solutions will be computed by the proposed method in conjunction with a stability analysis. Solutions will be compared to the results of temporal integrations, in showing excellent agreement.

\section{Harmonic balance method applied to a system with polynomial nonlinearities}

In this section, we will present an application of the harmonic balance method [5,19,3] for solving a nonlinear dynamic system along with the arc length continuation algorithms [6,5] introduced to follow the solution as frequency varies. In addition, the continuation procedure on the damping parameter for transforming an undamped solution into a damped solution will be presented.

\subsection{Harmonic balance method}

Let us consider an $n$ dof nonlinear dynamic system given by the following equation:

$$
\mathbf{M} \ddot{\boldsymbol{u}}+\mathbf{C} \boldsymbol{u}+\mathbf{K} \boldsymbol{u}+\boldsymbol{F}_{n l}(\boldsymbol{u})=\boldsymbol{F}(t)
$$

where $\boldsymbol{u}(t)$ is a vector of unknown size $n, \mathbf{M}, \mathbf{C}$ and $\mathbf{K}$ are respectively the mass, damping and stiffness matrices, $\boldsymbol{F}(t)$ is the excitation force vector which is assumed to be periodic with period $T=2 \pi / \omega$, and finally $\boldsymbol{F}_{n l}(\boldsymbol{u})$ is the vector of nonlinear forces assumed to be conservative and polynomial, i.e.,

$$
\left[\boldsymbol{F}_{n l}\right]_{i}(\boldsymbol{u})=\sum_{(\boldsymbol{\alpha}) \in \mathcal{S}_{i}} c(\boldsymbol{\alpha}) \boldsymbol{u}^{\boldsymbol{\alpha}}
$$

where $\mathcal{S}_{i} \subset \mathbb{N}^{n}$ is the support of polynomial $\left[\boldsymbol{F}_{n l}\right]_{i}$. Such dynamic systems arise, for example, after the finite element modeling of mechanical systems with geometric nonlinearity [3]. This paper focuses on the (possibly multiple) steady-state solutions of equation (1) under harmonic excitation. In order to compute this solution, the harmonic balance method (HBM) [19,5] is applied; this method consists of searching for the solution in the form of a truncated Fourier series up to the $H$ harmonic, as 
follows:

$$
\boldsymbol{u}(t)=\boldsymbol{a}_{0}+\sum_{k=1}^{H} \boldsymbol{a}_{k} \cos (k \omega t)+\boldsymbol{b}_{k} \sin (k \omega t)
$$

where $\boldsymbol{a}_{k}$ and $\boldsymbol{b}_{k}$ represent the Fourier coefficients associated with harmonic $k$. Next, Eq. (3) is substituted into Eq. (1) and the resulting equations are projected over the truncated Fourier basis $\boldsymbol{T}(t)=[1, \cos (\omega t), \sin (\omega t), \ldots, \cos (H \omega t), \sin (H \omega t)]$ using the scalar product $\langle f, g\rangle=(1 / T) \int_{0}^{T} f(t) g(t) d t$. The final result is a set of nonlinear algebraic equations given by the following equation:

$$
\mathbf{Z}(\omega) \boldsymbol{x}+\tilde{\boldsymbol{F}}_{n l}(\boldsymbol{x})-\tilde{\boldsymbol{F}}=\boldsymbol{P}(\boldsymbol{x}, \omega)=0
$$

where $\boldsymbol{x}=\left[\boldsymbol{a}_{0}^{T}, \boldsymbol{a}_{1}^{T}, \boldsymbol{b}_{1}^{T}, \ldots, \boldsymbol{a}_{H}^{T}, \boldsymbol{b}_{H}^{T}\right]^{T}$ is a vector of unknown size $n_{h}=n(2 H+1)$, and $\mathbf{Z}$ is the matrix of dynamic stiffness computed as $\mathbf{Z}=\operatorname{diag}\left(\mathbf{K},\left(\mathbf{Z}_{k}\right)_{1 \leq k \leq H}\right)$ where $\mathbf{Z}_{k}$ is given by

$$
\mathbf{Z}_{k}=\left[\begin{array}{cc}
\mathbf{K}-(k \omega)^{2} \mathbf{M} & k \omega \mathbf{C} \\
-k \omega \mathbf{C} & \mathbf{K}-(k \omega)^{2} \mathbf{M}
\end{array}\right]
$$

Moreover, $\tilde{\boldsymbol{F}}$ and $\tilde{\boldsymbol{F}}_{n l}(\boldsymbol{x})$ correspond respectively to the excitation force and the nonlinear force in the frequency domain. Since the nonlinear force in the time domain $\boldsymbol{F}_{n l}$ has been assumed to be polynomial, the resulting nonlinear force in the frequency domain $\tilde{\boldsymbol{F}}_{n l}$ is also in polynomial form, hence Eq. (4) is a system of $n_{h}$ polynomial equations. Fixing the frequency $\omega$ (e.g. as the excitation frequency (forced case) or the natural frequency (unforced case)) and solving the algebraic system in Eq. (4) then yields the possible steady states of the system for the particular frequency $\omega$.

\subsection{Continuation method}

A common way to solve Eq. (4) is to introduce continuation methods [6,5]. Starting from an initial point $\left(\boldsymbol{x}_{0}, \omega_{0}\right)$ solution of Eq. (4), a series of solutions $\left(\boldsymbol{x}_{i}, \omega_{i}\right)$ is derived through the use of a predictor/corrector scheme. The arc-length continuation is selected herein so that the solutions are parameterized by $s$, which represents a curvilinear abscissa. Then, starting from point $\left(\boldsymbol{x}_{i}, \omega_{i}\right)$, the next solution $\left(\boldsymbol{x}_{i+1}, \omega_{i+1}\right)$ is computed in two steps, as indicated in Fig. 1.

A predicted solution $\left(\boldsymbol{x}_{p}, \omega_{p}\right)=\left(\boldsymbol{x}_{i}+\Delta \boldsymbol{x}, \omega_{i}+\Delta \omega\right)$ is computed using a first-order Taylor expansion of Eq. (4) at point $i$ :

$$
\left[\partial_{\boldsymbol{x}} \boldsymbol{P}\left(\boldsymbol{x}_{i}, \omega_{i}\right)\right] \Delta \boldsymbol{x}+\left[\partial_{\omega} \boldsymbol{P}\left(\boldsymbol{x}_{i}, \omega_{i}\right)\right] \Delta \omega=0
$$

This equation is then complemented with another equation setting the length of the prediction:

$$
\|\Delta \boldsymbol{x}\|^{2}+|\Delta \omega|^{2}=d s^{2}
$$

where $d s$ is the step size. Combining Eqs. (6) and (7) leads to the following expressions for $\Delta \boldsymbol{x}$ and $\Delta \omega$ :

$$
\begin{aligned}
\Delta \omega & =\frac{ \pm d s}{\left(1+\left\|\left[\partial_{\boldsymbol{x}} \boldsymbol{P}\right]^{-1} \partial_{\omega} \boldsymbol{P}\right\|^{2}\right)^{1 / 2}} \\
\Delta \boldsymbol{x} & =-\left[\partial_{\boldsymbol{x}} \boldsymbol{P}\right]^{-1}\left[\partial_{\omega} \boldsymbol{P}\right] \Delta \omega
\end{aligned}
$$

The sign of $\Delta \omega$ is chosen according to the continuation direction (either forward or backward), in order to pass through turning points; $\Delta \omega$ should change sign in order to follow the curve. This sign change can be performed by monitoring the scalar product between two consecutive predictions: if it is negative, then $\Delta \omega$ changes sign.

Next, correction iterations are performed on the predicted point using the Newton-Raphson algorithm to solve the system in Eq. (9) for $\left(x_{i+1}, \omega_{i+1}\right)$ starting with initial iterate $\left(\boldsymbol{x}_{p}, \omega_{p}\right)$ :

$$
\begin{aligned}
& \boldsymbol{P}\left(\boldsymbol{x}_{i+1}, \omega_{i+1}\right)=0 \\
& \left\|\boldsymbol{x}_{i+1}-\boldsymbol{x}_{i}\right\|^{2}+\left|\omega_{i+1}-\omega_{i}\right|^{2}=d s^{2}
\end{aligned}
$$

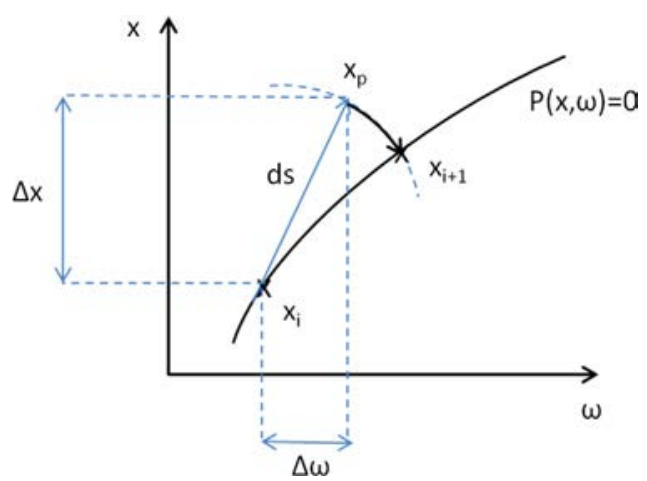

Fig. 1. Illustration of the arc length continuation procedure. 
The prediction/correction process is then applied to compute a desired number of points. The first point ( $\left.\boldsymbol{x}_{0}, \omega_{0}\right)$ is of particular interest since it determines which solution will be followed. Initial iterates $\boldsymbol{x}_{0}$ can be computed by solving Eq. (4) for a fixed frequency $\omega_{0}$ with the Newton-Raphson method using initial iterate $\boldsymbol{x}_{l}$, as given by the resolution of the linear system at the same frequency.

\subsection{Working on the undamped system}

As seen in both the Introduction and Section 3, Groebner basis computation time increases exponentially with the number of variables. Application of the HBM with $H$ harmonics on a system with $n$ dof results in $n_{h}=n(2 H+1)$ variables $($ or $n_{h}=2 H n$ if $\boldsymbol{a}_{\mathbf{0}}=0$ ). This number of variables can quickly become unworkable for the search of a Groebner basis within a reasonable time. To decrease the number of variables, we propose working on the undamped system, and search for inphase solutions by using only cosine terms in Eq. (3). This leads only to a subset of solutions (ie no travelling waves) but it has the advantage of reducing the number of variables from $n_{h}=(2 H+1) n$ to $n_{h}=(H+1) n\left(\right.$ or $n_{h}=n H$ if $\left.\boldsymbol{a}_{0}=0\right)$.

The in-phase solutions of the undamped problem will then be used as starting points for a damping continuation procedure, allowing to recover in-phase solutions of the damped problem. The continuation procedure on the damping parameter will be described hereafter.

In the undamped case, using only cosine terms, the HBM equation in Eq. (4) is reduced to the following equation:

$$
\mathbf{Z}(\omega) \boldsymbol{a}+\tilde{\boldsymbol{H}}(\boldsymbol{a})-\tilde{\boldsymbol{F}}=\boldsymbol{P}_{u}(\boldsymbol{a}, \omega)=0
$$

where $\mathbf{Z}=\operatorname{diag}\left(\mathbf{K},\left(\mathbf{K}-(k \omega)^{2} \mathbf{M}\right)_{1 \leq k \leq H}\right)$. Let us now suppose that all solutions $\boldsymbol{a}^{(m)}$ of the system in Eq. (10) have been found for a given frequency $\omega_{m}$; these solutions will now be used as a starting point for a continuation on the damping parameter. Replacing $\mathbf{C}$ by $\epsilon \mathbf{C}$ in Eq. (5) (where $\epsilon$ is a new parameter ranging from 0 to 1 ) results in the following form for Eq. (4):

$$
\mathbf{Z}(\omega, \epsilon) \boldsymbol{x}+\tilde{\boldsymbol{H}}(\boldsymbol{x})-\tilde{\boldsymbol{F}}=\boldsymbol{P}(\boldsymbol{x}, \omega, \epsilon)=0
$$

Let us note that since the nonlinearity is conservative, when $\epsilon=0$ the system is undamped and the vector $\boldsymbol{x}_{0}=\left[\boldsymbol{a}^{(m)}, \mathbf{0}\right]$ is a solution of Eq. (11); when $\epsilon=1$, the system is fully damped with matrix $\mathbf{C}$. A simple way to apply the continuation is to set $\omega$ at the fixed value $\omega_{m}$ and then apply a continuation on parameter $\epsilon$. However, as damping increases with $\epsilon$, we are not certain that a solution will always exist for this particular frequency (see, for example, Fig. 2 at $f=0.6 \mathrm{~Hz}$, solutions for $\epsilon=0.046$ exist, but not at $\epsilon=0.096$ ). To overcome this drawback, $\omega$ is also considered as a variable, and a continuation process with two parameters $\omega$ and $\epsilon$ based on a predictor/corrector is applied.

The prediction direction $\left(\boldsymbol{x}_{p}, \omega_{p}, \epsilon_{p}\right)=\left(\boldsymbol{x}_{i}+\Delta \boldsymbol{x}, \omega_{i}+\Delta \omega, \epsilon_{i}+\Delta \epsilon\right)$ is given by solving the following equation:

$$
\left[\partial_{\boldsymbol{x}} \boldsymbol{P}\left(\boldsymbol{x}_{i}, \omega_{i}, \epsilon_{i}\right)\right] \Delta \boldsymbol{x}+\left[\partial_{\omega} \boldsymbol{P}\left(\boldsymbol{x}_{i}, \omega_{i}, \epsilon_{i}\right)\right] \Delta \omega+\left[\partial_{\epsilon} \boldsymbol{P}\left(\boldsymbol{x}_{i}, \omega_{i}, \epsilon_{i}\right)\right] \Delta \epsilon=0
$$

This equation is underdetermined and should be complemented by two equations. The first equation imposes that the predicted vector must belong to the normal plane of the curve $\boldsymbol{P}\left(\boldsymbol{x}, \omega, \epsilon_{i}\right)=0$ at point $\left(x_{i}, \omega_{i}\right)$ :

$$
-\left(\left[\partial_{\boldsymbol{x}} \boldsymbol{P}\left(\boldsymbol{x}_{i}, \omega_{i}, \epsilon_{i}\right)\right]^{-1}\left[\partial_{\omega} \boldsymbol{P}\left(\boldsymbol{x}_{i}, \omega_{i}, \epsilon_{i}\right)\right]\right)^{T} \Delta \boldsymbol{x}+\Delta \omega=0
$$

The second equation sets the step length in the $\epsilon$ direction:

$$
\Delta \epsilon=\epsilon_{p}-\epsilon_{i}=d s
$$

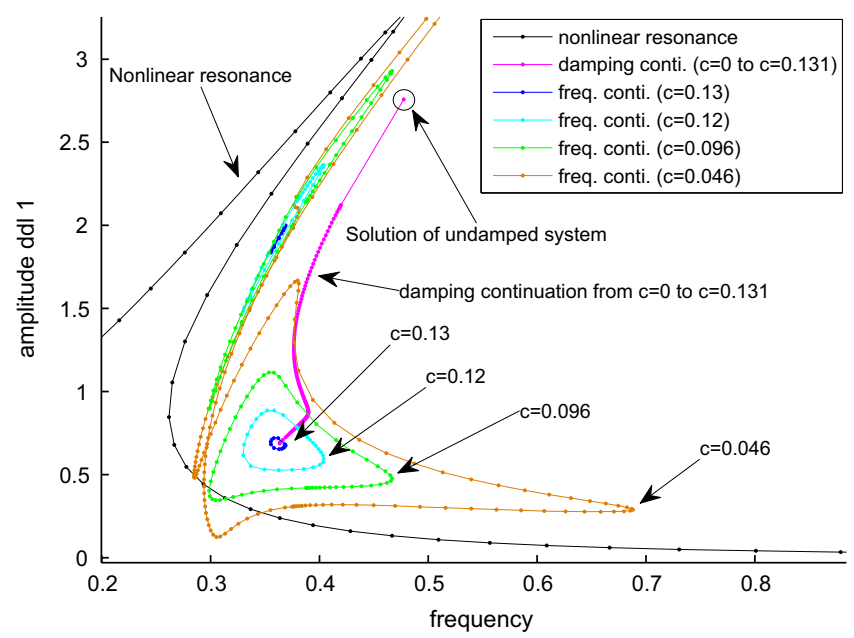

Fig. 2. Illustration of the continuation method on the damping parameter for the simple 2-dof system shown in Fig. 3 . 


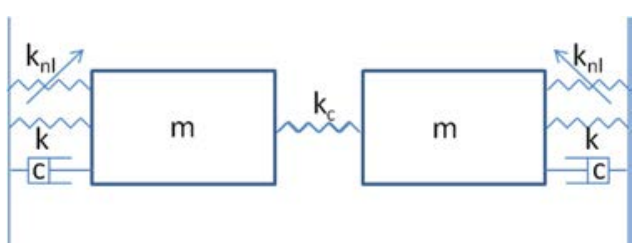

Fig. 3. Illustration of the simple 2-dof system.

Eqs. (12)-(14) can be combined to give the following expressions:

$$
\begin{aligned}
& \Delta \boldsymbol{x}=-\left(\left[\partial_{\boldsymbol{x}} \boldsymbol{P}\right]-\left[\partial_{\omega} \boldsymbol{P}\right]\left(\left[\partial_{\boldsymbol{x}} \boldsymbol{P}\right]^{-1}\left[\partial_{\omega} \boldsymbol{P}\right]\right)^{T}\right)^{-1}\left[\partial_{\epsilon} \boldsymbol{P}\right] \Delta \epsilon \\
& \Delta \omega=-\left(\left[\partial_{\boldsymbol{x}} \boldsymbol{P}\right]^{-1}\left[\partial_{\omega} \boldsymbol{P}\right]\right)^{T} \Delta \boldsymbol{x} \\
& \Delta \epsilon=d s
\end{aligned}
$$

Correction iterates are then applied to the predicted vector for $\epsilon=\epsilon_{p}$. The Newton-Raphson method is used to solve the following system with initial iterate $\left(\boldsymbol{x}_{p}, \omega_{p}\right)$ :

$$
\begin{aligned}
& \epsilon_{i+1}-\epsilon_{p}=0 \\
& \boldsymbol{P}\left(\boldsymbol{x}_{i+1}, \omega_{i+1}, \epsilon_{i+1}\right)=0 \\
& \left(\left[\partial_{\boldsymbol{x}} \boldsymbol{P}\right]^{-1}\left[\partial_{\omega} \boldsymbol{P}\right]\right)^{T}\left(\boldsymbol{x}_{i+1}-\boldsymbol{x}_{i}\right)+\left(\omega_{i+1}-\omega_{i}\right)=0
\end{aligned}
$$

The last equation of the system in Eq. (16) corresponds to the fact that correction points should lie on the normal plane, as defined in the prediction step (Eq. (13)).

The prediction/correction process is then applied until $\epsilon=1$, providing a solution $\left(\boldsymbol{x}^{(m)}, \omega^{(m)}\right)$ of the damped system. Moreover, the continuation is repeated for all starting points $\boldsymbol{x}_{0}=\left[\boldsymbol{a}^{(m)}, \mathbf{0}\right]$.

Remarks. For some starting points, the solution may disappear as damping increases, thus leading to an unfinished continuation. This fact is exemplified in Fig. 2, where the closed curve solution is seen to shrink as damping increases. As a byproduct of this continuation method, the maximum damping coefficient $\epsilon$ is obtained in order for a solution to exist. For instance, let us consider the 2 dof example depicted in Fig. 3. The motion equations are given by the following equations:

$$
\begin{aligned}
& m \ddot{u}_{1}+c \dot{u}_{1}+\left(k+k_{c}\right) u_{1}-k_{c} u_{2}+k_{n l} u_{1}^{3}=f \cos (\omega t) \\
& m \ddot{u}_{2}+c \dot{u}_{2}+\left(k+k_{c}\right) u_{2}-k_{c} u_{1}+k_{n l} u_{2}^{3}=f \cos (\omega t)
\end{aligned}
$$

with $k=k_{c}=m=k_{n l}=c=1$. We now introduce the damping continuation parameter $\epsilon$ by replacing $c$ by $\epsilon c=\epsilon \times 1=\epsilon$ in Eq. (17), so that the new damping coefficient is directly driven by $\epsilon$. Fig. 2 shows that when $\epsilon \geq 0.131$, the closed curve solution no longer exists; therefore, the maximum damping coefficient for this closed curve solution to exist is given by $\epsilon=0.131$.

\section{Multiple steady-state solutions of the dynamic system}

Following a short review of existing methods for finding multiple solutions of nonlinear dynamic systems, this section will focus on the use of Groebner bases for solving polynomial systems.

\subsection{Review}

The methods for identifying multiple steady-state solutions of a dynamic system can be classified into two categories: methods in the time domain and methods in the frequency domain. Time domain methods, such as the cell mapping method [20,21], directly deal with the system of differential equations. Most of the time, those methods give plenty of information on the system (solutions, stability, basin of attraction, etc.), but they are often limited to very small systems due to the computation times required.

Here, we will concentrate on frequency domain methods that first transform the set of differential equations in a set of algebraic equations. In the case of polynomial nonlinearities, the search for multiple steady-state solutions is most of the time reduced to finding multiple solutions of a multivariate polynomial system. Finding all solutions of a multivariate polynomial system is a topic of considerable interest since systems of polynomial equations are encountered in many research fields. In most instances, the system is solved by means of Newton-Raphson algorithms [6], which only yield one solution depending on the initial iterate. The Newton-Raphson algorithm is typically coupled with continuation methods in order to follow the solution [6] as frequency varies. In some cases, bifurcation occurs due to special values of the parameter $\omega$. Depending on the bifurcation type, new branches of solutions can be computed from the bifurcation points [6].

Homotopy methods offer an alternative that can actually compute all solutions of polynomial equation systems. Linear homotopy [3] relies on linking the root of the system $\boldsymbol{P}$ to the roots of a simpler system $\boldsymbol{Q}$ via a regular path. The roots of systems $\boldsymbol{Q}$ are known while the roots of system $\boldsymbol{P}$ are computed by following the paths given by $\boldsymbol{H}(\boldsymbol{x}, t)=t \boldsymbol{P}(\boldsymbol{x})+(1-t) \boldsymbol{Q}(\boldsymbol{x})$ through continuation methods on parameter $t$. System $\boldsymbol{Q}$ generally contains more roots than system $\boldsymbol{P}$, hence some paths 
will diverge and their continuation will only result in a loss of time. Polyhedral homotopy [10] is aimed at solving this problem by the use of the so-called "BKK bound" [8,9], which allows to construct an initial polynomial $\boldsymbol{Q}$ which has the same number of roots as $\boldsymbol{P}$ (almost surely). The construction of the polyhedral homotopie relies however on probabilistic arguments and many combinatorial problems, making it very complex and time consuming.

Algebraic methods such as Groebner basis [11], rational univariate representation [12,13] and multiplication matrix [14-16] are another alternative for solving polynomial systems. These methods exploit the relationship between variables derived by the set of polynomial equations, and they are mainly based on Groebner bases. As seen in the next section, the choice to use a Groebner basis for solving multivariate polynomials is motivated by the fact that they allow finding multiple solutions of multivariate polynomials in a relatively simple way: by either solving a univariate polynomial or computing eigenvalues of special matrices.

\subsection{Groebner basis and multivariate polynomial solutions}

This section will present the use of Groebner basis to find all solutions of a multivariate polynomial system $\boldsymbol{P}(\boldsymbol{x})=\mathbf{0}$. But, before introducing Groebner basis, we recall some definitions related to systems of multivariate polynomial equations.

We denote $\mathbb{C}[\boldsymbol{x}]$ the ring of multivariate polynomials with complex coefficients defined by the following:

$$
\mathbb{C}[\boldsymbol{x}]=\left\{f \mid f(\boldsymbol{x})=\sum_{\boldsymbol{\alpha} \in \mathcal{S}} c(\boldsymbol{\alpha}) \boldsymbol{x}^{\boldsymbol{\alpha}}, c(\boldsymbol{\alpha}) \in \mathbb{C}\right\}
$$

$\mathcal{S} \subset \mathbb{N}^{n}$ is called the support of polynomials $f, \boldsymbol{x}^{\boldsymbol{\alpha}}=x_{1}^{\alpha_{1}} \ldots x_{n}^{\alpha_{n}}$ is called a monomial of total degree $|\boldsymbol{\alpha}|=\sum_{i} \alpha_{i}$, and $c(\boldsymbol{\alpha}) \in \mathbb{C}$ is the coefficient of monomial $\boldsymbol{x}^{\alpha}$. As monomials can be seen as points in $\mathbb{N}^{n}$, multiple monomial ordering can be defined. Two kinds of ordering are generally used: (i) lexicographic ordering $\left(\leq_{l e x}\right)$ and (ii) graded reverse lexicographic ordering ( $\leq$ grev).

For $(\boldsymbol{\alpha}, \boldsymbol{\beta}) \in\left[\mathbb{N}^{n}\right]^{2}$, they are respectively defined by

$$
\boldsymbol{\alpha} \leq_{l e x} \boldsymbol{\beta} \equiv\left[\alpha_{j} \leq \beta_{j} \text { and } \alpha_{i}=\beta_{i} \text { for } 1 \leq i \leq j \leq n\right]
$$

and

$$
\boldsymbol{\alpha} \leq \text { grev } \boldsymbol{\beta} \equiv[|\boldsymbol{\alpha}| \leq|\boldsymbol{\beta}|] \quad \text { or } \quad\left[\alpha_{j} \geq \beta_{j} \text { and } \alpha_{i}=\beta_{i} \text { for } 1 \leq j \leq i\right]
$$

We denote $\mathcal{I}$ (or equivalently $\langle\boldsymbol{P}\rangle$ ) the ideal of $\mathbb{C}[\boldsymbol{x}]$ generated by the polynomial system $\boldsymbol{P}$ and defined by

$$
\mathcal{I}=\left\{f \in \mathcal{C}[\boldsymbol{x}] f(\boldsymbol{x})=\sum_{i=1}^{n} \mu_{i}(\boldsymbol{x}) p_{i}(\boldsymbol{x}), \mu_{i} \in \mathbb{C}[\boldsymbol{x}]\right\}
$$

Finally, we denote $\rightarrow \boldsymbol{P}$ the reduction operation modulo $\mathcal{I}$ (or equivalently modulo $\boldsymbol{P}$ ) defined for $f \in \mathbb{C}[\boldsymbol{x}]$ by $f \rightarrow \boldsymbol{P} r(f$ reduces to $r$ modulo $\boldsymbol{P}$ ) where $r \in \mathbb{C}[\boldsymbol{x}]$ is defined by the following relation:

$$
f(\boldsymbol{x})=\sum_{i=1}^{n} \mu_{i}(\boldsymbol{x}) p_{i}(\boldsymbol{x})+r(\boldsymbol{x})
$$

In other words $r$ is the remainder in the division of $f$ by each element of $\boldsymbol{P}$. This remainder is generally not unique and depends on the division order and on the monomial order.

Let us now consider a polynomial system $\boldsymbol{P}(\boldsymbol{x})$ generating an ideal $\mathcal{I}=\langle\boldsymbol{P}\rangle$. The concept of Groebner basis for polynomial ideals was introduced in the 1960s by Buchberger and Groebner [22]. A Groebner basis $\boldsymbol{G}$ for an ideal $\mathcal{I}$ relatively to a monomial order can be defined as a polynomial system $\boldsymbol{G}$ that generates the ideal $\mathcal{I}$ (i.e $\mathcal{I}=\langle\boldsymbol{G}\rangle$ ), and that make the remainder unique in the reduction operation $\rightarrow_{\boldsymbol{G}}$ (for a fixed monomial order).

Formally, $\boldsymbol{G}=\left[g_{1}, \ldots, g_{r}\right]$ is a Groebner basis for $\mathcal{I}$ if the leading term of each element of $\mathcal{I}$ can be divided by the leading term of a polynomial $g_{i}$ for a particular $i \in\{1, \ldots, r\}$.

Buchberger proposed an algorithm for computing Groebner bases in his thesis [22] that has been improved several times since, in particular by Faugère [23-25]. Note that the monomial ordering used in Groebner basis computations influences both form of the basis $\boldsymbol{G}$ and computation time, and, in general, computation of Groebner basis using the grevlex ordering is faster than with the lexicographic ordering.

\subsubsection{Groebner basis with elimination order}

The fact that a Groebner basis generates the same ideal as $\boldsymbol{P}$ implies that the system $\boldsymbol{G}(\boldsymbol{x})=\mathbf{0}$ has the same solutions as the system $\boldsymbol{P}(\boldsymbol{x})=\mathbf{0}$. For some particular monomial ordering, the associated Groebner basis $\boldsymbol{G}$ can have a simpler form than $\boldsymbol{P}$, and thus can be solved more easily. Using the so-called "elimination order" (such as lexicographic ordering [26]) allows computing a Groebner basis $\boldsymbol{G}$ with a special triangular form, as given in the following equation:

$$
\begin{aligned}
& g_{1}\left(x_{1}, \ldots, x_{n}\right)=0 \\
& \vdots \\
& g_{r-1}\left(x_{n-1}, x_{n}\right)=0 \\
& g_{r}\left(x_{n}\right)=0
\end{aligned}
$$


This particular form of Groebner basis can be directly used to find the zeros of $\boldsymbol{P}$. The last equation is indeed a univariate polynomial equation, which can be solved efficiently, yielding values for $x_{n}$. The values of $x_{n-1}$ are then computed using the penultimate equation, and the lifting sequence continues until the values for $x_{1}$ are computed.

To illustrate the special form of Groebner basis computed relative to an elimination order, let us consider a simple twovariate polynomial system $\boldsymbol{P}\left(x_{1}, x_{2}\right)$ derived from Eq. (4) for a 2-dof system depicted in Fig. 3 and given by the following equation:

$$
\boldsymbol{P}\left(x_{1}, x_{2}\right)=\left[\begin{array}{cc}
2-\omega^{2} & -1 \\
-1 & 2-\omega^{2}
\end{array}\right]\left[\begin{array}{l}
x_{1} \\
x_{2}
\end{array}\right]+\frac{3}{4}\left[\begin{array}{l}
x_{1}^{3} \\
x_{2}^{3}
\end{array}\right]-\left[\begin{array}{l}
1 \\
1
\end{array}\right]
$$

where $\omega$ is a parameter and $m=k=k_{c}=k_{n l}=f=1$.

Computing a Groebner basis $\boldsymbol{G}^{\text {lex }}$ for the system in Eq. (24) relative to the lexicographic ordering with $x_{1}>x_{2}$ results in the following expression for $\boldsymbol{G}^{\text {lex }}=\left[g_{1}, g_{2}\right]$ :

$$
\begin{aligned}
& \boldsymbol{G}_{e x, 1}^{(\text {lex })}\left(\boldsymbol{x}_{1}, \boldsymbol{x}_{2}\right)=-4-4 \boldsymbol{x}_{1}+\left(8-4 \omega^{2}\right) \boldsymbol{x}_{2}+3 \boldsymbol{x}_{2}^{3} \\
& \boldsymbol{G}_{e x, 2}^{(\text {lex) }}\left(\boldsymbol{x}_{2}\right)=h_{0}(\omega)+h_{1}(\omega) \boldsymbol{x}_{2}+h_{2}(\omega) \boldsymbol{x}_{2}^{2}+h_{3}(\omega) \boldsymbol{x}_{2}^{3}+h_{4}(\omega) \boldsymbol{x}_{2}^{4}+h_{5}(\omega) \boldsymbol{x}_{2}^{5}-h_{6}(\omega) \boldsymbol{x}_{2}^{6}+h_{7}(\omega) \boldsymbol{x}_{2}^{7}+81 \boldsymbol{x}_{2}^{9}
\end{aligned}
$$

where the functions $h_{i}(\omega)$ are univariate polynomials of maximum degree 4 (for this special case) with variable $\omega$. For this unique application, let us note that the second equation of the Groebner basis in Eq. (25) is always of degree 9 and therefore has 9 complex solutions. Solving the last equation and substituting the results into the first equation leads to a final set of 9 complex solutions $\left(\boldsymbol{x}_{1}, \boldsymbol{x}_{2}\right)$ for Eq. (24).

The main drawback of computing a Groebner basis with an elimination order is the exponential increase in computation time with the number of variables. To overcome this drawback, it is possible to compute a Groebner basis $\boldsymbol{G}^{\text {lex }}$ in less time by using another order, such as the graded reverse lexicographic ordering (grevlex), which seems to offer the least computation time [27], and then recasting the basis $\boldsymbol{G}^{\text {grev }}$ into a triangular one $\boldsymbol{G}^{\text {lex }}$ using the FGLM algorithm [23]. However, the computation time of FGLM algorithms increases considerably with the number of variables. To overcome these drawbacks, we propose introducing a method that directly outputs the solution of the polynomial system by means of an eigenvector computation.

\subsubsection{Multiplication matrix method}

Another particular feature of Groebner basis is the fact that the remainder $r$ in the reduction of $f$ modulo $\mathcal{I}\left(f \rightarrow{ }_{G} r\right)$ is unique. This allows to extend the concept of Euclidean division for multivariate polynomials and allows calculating in the quotient space $\mathbb{Q}=\mathbb{C}[\boldsymbol{x}] / \mathcal{I}$. $\mathbb{Q}$ contains all remainders $r$ (also called normal forms) that may appear in a reduction modulo $\mathcal{I}$, and for $q_{1}, q_{2} \in \mathbb{Q}$ we define the equivalence relation:

$$
\left[q_{1}=q_{2}\right] \equiv\left[q_{1}-q_{2} \in \mathcal{I}\right]
$$

In the case where the polynomial system $\boldsymbol{P}(\boldsymbol{x})=\mathbf{0}$ has only a finite number of solutions (say $D$ solutions), it can be shown [14-16] that $\mathbb{Q}$ is a vectorial space of finite dimension $D$. Groebner basis can be used to compute a (monomial) basis $\mathcal{B}$ for $\mathbb{Q}$ as a vectorial space on $\mathbb{C}$ (the basis $\mathcal{B}$ is also called a normal set). The normal set $\mathcal{B}$ may be viewed as the set of all monomials capable of appearing in a remainder $r$ in the reduction modulo $\mathcal{I}$ (i.e each $r$ can be decomposed as $r(\boldsymbol{x})=\sum_{j=1}^{D} c_{j} \mathcal{B}_{j}(\boldsymbol{x}), c_{j} \in \mathbb{C}$ ), and it can be formally defined as follows:

$$
\mathcal{B}=\left\{\boldsymbol{x}^{\boldsymbol{\alpha}} \mid \boldsymbol{x}^{\alpha} \notin\langle L T(\boldsymbol{G})\rangle\right\}
$$

where $\langle L T(\boldsymbol{G})\rangle$ is the ideal generated by the leading terms of $\boldsymbol{G}$.

Since the quotient space $\mathbb{Q}$ is a vector space of finite dimension, all linear applications can be represented by a matrix relative to basis $\mathcal{B}$. A particular linear application set is the application " $m_{f}$ : multiplication by polynomial $f \in \mathbb{C}[\boldsymbol{x}]$ " defined as

$$
\begin{aligned}
& m_{f}: \mathbb{C}[\boldsymbol{x}] / \mathcal{I} \rightarrow \mathbb{C}[\boldsymbol{x}] / \mathcal{I} \\
& h \mapsto m_{f}(h)=f h
\end{aligned}
$$

The matrices $\mathbf{M}_{f}$ associated with applications $m_{f}$ relative to basis $\mathcal{B}$ are called multiplication matrices, and they can be characterize by the following relation (modulo $\mathcal{I}$ ):

$$
f \mathcal{B}=\mathbf{M}_{f} \mathcal{B}
$$

or equivalently

$$
f \mathcal{B}_{i}=\sum_{j=1}^{D} M_{i, j}^{f} \mathcal{B}_{j}
$$

For particular choices of $f=x_{i}, i=1, \ldots, n$, it can be shown [14-16] that the eigenvalues of the multiplication matrices $\mathbf{M}_{x_{i}}$ are related to the zeros of the polynomial system. Indeed, the values $x_{i}^{(k)}$ of variables $x_{i}$ for solution $k$ are given by the eigenvalues of $\mathbf{M}_{x_{i}}$ associated with common eigenvector $\boldsymbol{V}_{k}$. Component $x_{i}^{(k)}$ of solution $k$ can then be obtained directly by reading the eigenvector $\boldsymbol{V}_{k}$ at location $j$, where $j$ is the location of monomial $x_{i}$ in the quotient space basis $\mathcal{B}$ (note: the eigenvectors should be normalized so that their first component [associated with monomial $\boldsymbol{x}^{\mathbf{0}}=1$ ] equals one). 
To avoid computing the eigenvectors of all multiplication matrices $\mathbf{M}_{x_{i}} i=1, \ldots, n$, we only consider the multiplication matrix associated with the linear combination $f=\sum_{i=1}^{n} c_{i} x_{i}$, where $c_{i}$ are rational numbers chosen such that the values $f\left(\boldsymbol{x}^{(k)}\right)$ are different at each solutions points $\boldsymbol{x}^{(k)}, k=1, \ldots, D$. Generally, random choices for coefficients $c_{i}$ are sufficient to ensure these properties almost surely [28]. The eigenvalues and eigenvectors of $\mathbf{M}_{f}$ are then computed and the component of the solutions can be read in the eigenvector, as previously described.

In general, the computation of basis $\mathcal{B}$ and the construction of multiplication matrices require greater amounts of time as the number of variables increases. Some symbolic computation software already feature packages relative to Groebner basis computations, such as the 'Groebner' package of the Maple software. However, for certain special cases of polynomial systems, the Groebner basis $\boldsymbol{G}$ and monomial basis $\mathcal{B}$ can be quickly determined (e.g. see Section 4).

In sum, the resolution process used in the multiplication matrix method to solve a polynomial system $\boldsymbol{P}$ is the following:

- Compute a Groebner basis $\boldsymbol{G}$ relative to the grevlex order (fastest order).

- Use this Groebner basis $\boldsymbol{G}$ to compute a normal set $\mathcal{B}$ for the quotient space $\mathbb{Q}$ (use of the reduction operation).

- Choose $f=\sum c_{i} x_{i}$ and build the multiplication matrix $\mathbf{M}_{f}$ relative to basis $\mathcal{B}$.

- Compute and normalize the eigenvectors of the multiplication matrix.

- Read the solution components $x_{i}^{(k)}, i=1, \ldots, n$ in the eigenvector $\boldsymbol{V}_{k}$, for $k=1, \ldots, D$.

As an application, we computed a normal set $\mathcal{B}$ for the quotient space $\mathbb{Q}$ induced by the polynomial system in Eq. (24). We began by computing a Groebner basis $\boldsymbol{G}^{\text {(grev) }}$ relative to the graded reverse lexicographic ordering (grevlex). It turns out that the system in Eq. (24) is already in Groebner basis form for the grevlex ordering, hence $\boldsymbol{G}^{\text {(grev) }}=\boldsymbol{P}$. The ideal generated by the leading terms of the Groebner basis is given by $\left\langle\operatorname{LT}\left(\boldsymbol{G}^{(\text {grev })}\right)\right\rangle=\left\langle\boldsymbol{x}_{\mathbf{1}}^{3}, \boldsymbol{x}_{2}^{3}\right\rangle$ and therefore basis $\mathcal{B}=\left\{\boldsymbol{x}^{\boldsymbol{\alpha}}, \boldsymbol{\alpha} \in[0,2]^{2}\right\}($ see definition in Eq. (27)) which leads to

$$
\mathcal{B}=\left[1, x_{1}, x_{2}, x_{1}^{2}, x_{1} x_{2}, x_{2}^{2}, x_{1}^{2} x_{2}, x_{1} x_{2}^{2}, x_{1}^{2} x_{2}^{2}\right]
$$

Let us note that in this special case, basis $\mathcal{B}$ contains 9 elements and therefore $\mathbb{Q}$ is of dimension 9 , and the system in Eq. ( 24 ) has 9 solutions.

Now that we have a basis for $\mathbb{Q}$, we can compute the multiplication matrix associated to $f=x_{1}+\frac{2}{3} x_{2}$ relative to basis $\mathcal{B}$. To illustrate the process, we show how to compute the first and the last line of the multiplication matrix. The first line is associated with the product $f \mathcal{B}_{1}$ which can be developed as

$$
f \mathcal{B}_{1}=\left(x_{1}+\frac{2}{3} x_{2}\right) \times 1=\underbrace{1}_{\mathbf{M}_{1,2}^{f}} \times \mathcal{B}_{2}+\underbrace{\frac{2}{3}}_{\mathbf{M}_{1,3}^{f}} \times \mathcal{B}_{3}
$$

Using this last equation we can see that the product $f \mathcal{B}_{1}$ can directly be expressed in terms of elements of $\mathcal{B}$, so that the first line of the multiplication matrix is directly identified and will contain a 1 in position $(1,2)$, a $\frac{2}{3}$ in position (1, 3) and zeros elsewhere. The last line of the matrix is associated with the product $f \mathcal{B}_{9}$ which can be developed as

$$
f \mathcal{B}_{9}=\left(x_{1}+\frac{2}{3} x_{2}\right) \times x_{1}^{2} x_{2}^{2}=x_{1}^{3} x_{2}^{2}+\frac{2}{3} x_{1}^{2} x_{2}^{3}
$$

Here we can see that the product $f \mathcal{B}_{9}$ contains terms of degree 5 , whereas the basis $\mathcal{B}$ has term of degree at most 4 . In this case we have to compute the normal form of $f \mathcal{B}_{9}$ using the reduction operation relative to Groebner basis $\boldsymbol{G}^{\text {grev }}$ computed previously. In this case the reduction operation gives $f \mathcal{B}_{9} \rightarrow G^{\text {grev }} r$ with

$$
r=\underbrace{\frac{80}{27}}_{\mathbf{M}_{9,1}}+\underbrace{\frac{4}{27}\left(-4+8 \omega^{2}\right)}_{\mathbf{M}_{9,2}^{f}} x_{1}+\underbrace{\frac{4}{27}\left(-16+12 \omega^{2}\right)}_{\mathbf{M}_{9,3}^{f}} x_{2}+\underbrace{\frac{4}{27}\left(-12+6 \omega^{2}\right)}_{\mathbf{M}_{9,7}^{f}} x_{1}^{2} x_{2}+\underbrace{\frac{4}{27}\left(-18+9 \omega^{2}\right)}_{\mathbf{M}_{9,8}^{f}} x_{2}^{2} x_{1}
$$

Using the same procedure for all products $f \mathcal{B}_{i}, i=1, \ldots, D$, results in the following expression for $\mathbf{M}_{f}$ :

$$
\mathbf{M}_{f}=\left[\begin{array}{ccccccccc}
0 & 1 & \frac{2}{3} & 0 & 0 & 0 & 0 & 0 & 0 \\
0 & 0 & 0 & 1 & \frac{2}{3} & 0 & 0 & 0 & 0 \\
0 & 0 & 0 & 0 & 1 & \frac{2}{3} & 0 & 0 & 0 \\
\frac{4}{3} & -\frac{8}{3}+\frac{4}{3} \omega^{2} & \frac{4}{3} & 0 & 0 & 0 & \frac{2}{3} & 0 & 0 \\
0 & 0 & 0 & 0 & 0 & 0 & 1 & \frac{2}{3} & 0 \\
\frac{8}{9} & \frac{8}{9} & -\frac{16}{9}+\frac{8}{9} \omega^{2} & 0 & 0 & 0 & 0 & 1 & 0 \\
0 & 0 & 0 & 0 & -\frac{8}{3}+\frac{4}{3} \omega^{2} & \frac{4}{3} & 0 & 0 & \frac{2}{3} \\
0 & 0 & 0 & \frac{8}{9} & -\frac{16}{9}+\frac{8}{9} \omega^{2} & 0 & 0 & 0 & 1 \\
\frac{80}{27} & -\frac{16}{27}+\frac{32}{27} \omega^{2} & -\frac{64}{27}+\frac{16}{9} \omega^{2} & 0 & 0 & 0 & -\frac{16}{9}+\frac{8}{9} \omega^{2} & -\frac{8}{3}+\frac{4}{3} \omega^{2} & 0
\end{array}\right]
$$

After computing the (normalized) eigenvectors of matrix $\mathbf{M}_{f}$ the solution $\left(x_{1}, x_{2}\right)^{(k)}$ of $\boldsymbol{P}\left(x_{1}, x_{2}\right)=\mathbf{0}$ will be read as $\left(V_{2}^{(k)}, V_{3}^{(k)}\right)$ (where $V_{i}^{(k)}$ is the $i$-th component of eigenvector $\boldsymbol{V}^{(k)}$ ). 


\section{Application to a numerical example}

\subsection{Simple 2 dof system}

As a first application, we consider a simple undamped, unforced 2-dof nonlinear system depicted in Fig. 3. The goal of this example is to illustrate the search for multiple solutions using the multiplication matrix method, and to see the convergence of the solution as the number of retained harmonics increases. The motion equation for such a system can be given by the following equation:

$$
\begin{aligned}
& m \ddot{u}_{1}+\left(k+k_{c}\right) u_{1}-k_{c} u_{2}+k_{n l} u_{1}^{3}=0 \\
& m \ddot{u}_{2}+\left(k+k_{c}\right) u_{2}-k_{c} u_{1}+k_{n l} u_{2}^{3}=0
\end{aligned}
$$

In the numerical application we will set $m=k=k_{c}=k_{n l}=1$.

In order to compute an approximated solution of system (36), the harmonic balance is used. As no static forces act on the system and as the nonlinearity is purely cubic, no continuous component will be retrained in the HBM development (i.e $\left.\boldsymbol{a}_{0}=0\right)$. In addition, a maximum of 3 harmonics will be used, so that the solutions $u_{1}(t)$ and $u_{2}(t)$ will be expressed as

$$
\begin{aligned}
& u_{1}(t)=\sum_{k=1}^{H} x_{i} \cos (k \omega t) \\
& u_{2}(t)=\sum_{k=1}^{H} y_{i} \cos (k \omega t)
\end{aligned}
$$

In order to evaluate the convergence of the solutions with the number of retained harmonics $H$, three different levels of truncation will be considered, going from $H=1$ to $H=3$. The nonlinear algebraic system arising from application of the HBM with $H=3$ harmonics $\left(n_{H}=6\right)$ can be written as follows:

$$
\begin{aligned}
& \left(2-\omega^{2}\right) x_{1}-y_{1}+\frac{3}{2} x_{1}\left(x_{2}^{2}+x_{3}^{2}\right)+\frac{3}{4}\left(x_{1}^{3}+x_{1}^{2} x_{3}+x_{2}^{2} x_{3}\right)=0 \\
& \left(2-\omega^{2}\right) y_{1}-x_{1}+\frac{3}{2} y_{1}\left(y_{2}^{2}+y_{3}^{2}\right)+\frac{3}{4}\left(y_{1}^{3}+y_{1}^{2} y_{3}+y_{2}^{2} y_{3}\right)=0 \\
& \left(2-4 \omega^{2}\right) x_{2}-y_{2}+\frac{3}{2} x_{2}\left(x_{1}^{2}+x_{3}^{2}+x_{1} x_{3}\right)+\frac{3}{4} x_{2}^{3}=0 \\
& \left(2-4 \omega^{2}\right) y_{2}-x_{2}+\frac{3}{2} y_{2}\left(y_{1}^{2}+y_{3}^{2}+y_{1} y_{3}\right)+\frac{3}{4} y_{2}^{3}=0 \\
& \left(2-9 \omega^{2}\right) x_{3}-y_{3}+\frac{3}{2} x_{3}\left(x_{2}^{2}+x_{1}^{2}\right)+\frac{3}{4}\left(x_{3}^{3}+x_{1} x_{2}^{2}\right)=0 \\
& \left(2-9 \omega^{2}\right) y_{3}-x_{3}+\frac{3}{2} y_{3}\left(y_{2}^{2}+y_{1}^{2}\right)+\frac{3}{4}\left(y_{3}^{3}+y_{1} y_{2}^{2}\right)=0
\end{aligned}
$$

The system for $H=1$ [resp. $H=2$ ] can be obtained by setting $x_{2}=y_{2}=x_{3}=y_{3}=0$ [resp. $x_{3}=y_{3}=0$ ] and by retaining only the two [resp. the four] first equations.

At first, we set $\omega=\frac{25}{10}$ and we solve Eq. (38) with the multiplication matrix method for $H=1,2,3$. The results are summarized in Table 1 and solutions are depicted in Figs. 4-6. In order to simplify the analysis, we sorted the solution by family by using the symmetry of the algebraic system (38). Indeed, it can be seen that this system is invariant under the application $\mathcal{T}_{c}:\left(x_{i}, y_{i}\right) \mapsto\left(y_{i}, x_{i}\right), i=1,2,3$ (change of coordinate), and $\mathcal{T}_{s}:\left(x_{i}, y_{i}\right) \mapsto\left(-x_{i},-y_{i}\right), i=1,2,3$ (change of sign). Therefore, two solutions $\boldsymbol{s}_{1}$ and $\boldsymbol{s}_{2}$ will be part of the same family if $\boldsymbol{s}_{1}=\mathcal{T}\left(\boldsymbol{s}_{2}\right)$, where $\mathcal{T}=\mathcal{T}_{s}$ or $\mathcal{T}=\mathcal{T}_{c}$.

For $H=1$ harmonics, only 3 families of solution ( +1 trivial solution) are obtained (Fig. 4 ). They correspond respectively to the first NNM (NNM1, form [a,a], in phase), the second NNM (NNM2, form $[b,-b]$ out of phase) and a bifurcation of the second mode which corresponds to a localized solution (NNMloc, form $[c,-d]$, out of phase). When we increase the number of harmonics, these 3 families of solution remain, and we observe the apparition of new solutions: (i) subharmonic oscillations and (ii) internal resonances. For $H=2$ we detect 3 families of solution corresponding to subharmonic oscillations of order 2 (directly related to the NNMs), and two internal resonances of type 1:2 (one coordinate vibrate at $\omega$ and the other at $2 \omega$, Fig. 4). For $H=3$, subharmonics of order 3 are observed along with 4 internal resonances: 2 of type $1: 3$ and 2 of type $2: 3$, Fig. 6 . We can see that each internal resonances can either be in a mode 1 fashion (each amplitude is positive) or in a mode 2 fashion (one amplitude positive and one negative).

For $H=3$ harmonics, we use the computed solutions as starting points for an arc-length continuation. Results are depicted in Fig. 7 showing NNM1, NNM2, NNMloc and their subharmonic oscillations. Fig. 8 shows the internal resonances curves. The subharmonic oscillations can directly be obtained from mode 1 , mode 2 and the localized mode simply by dividing the frequency by 2 (order 2 ) or by 3 (order 3 ).

This example shows that even extremely simple systems can exhibit a very complex dynamic behavior. The use of the HBM in addition with the multiplication matrix method allows to find a great number of solutions which can be sorted by family. When increasing the number of retained harmonics, all previously computed solutions remain, and some more solutions are detected such as subharmonic oscillations or internal resonances.

This first example was very simple and considered undamped. We will now turn to the case of a more complex structure possessing cyclic symmetry, in order to apply the multiplication matrix method in addition with the damping continuation method presented in Section 2.3. 
Table 1

Results of the multiplication matrix method applied to systems (38) for $H=1,2,3$ harmonics.

\begin{tabular}{llll}
\hline Number of harm. $H$ & 1 & 2 & 3 \\
\hline Number of sol. $D$ & 9 & 81 & 729 \\
Number of real sol. & 9 & 25 & 49 \\
Number of family & $3(+1)$ & $8(+1)$ & $15(+1)$ \\
\hline
\end{tabular}

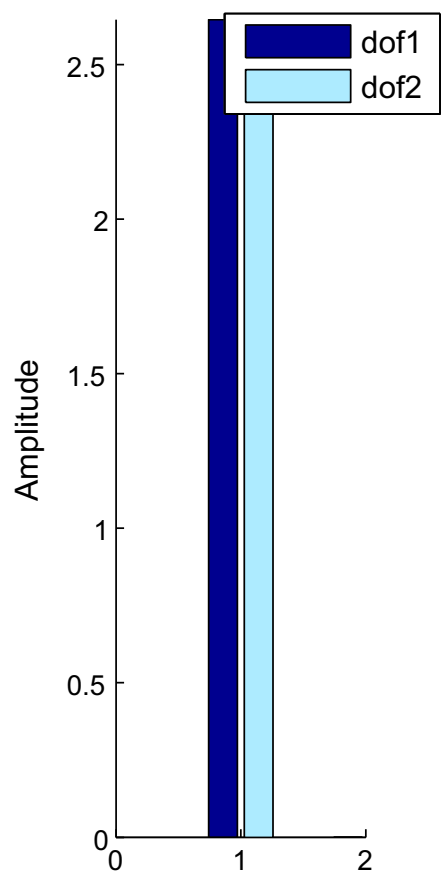

Harmonic number

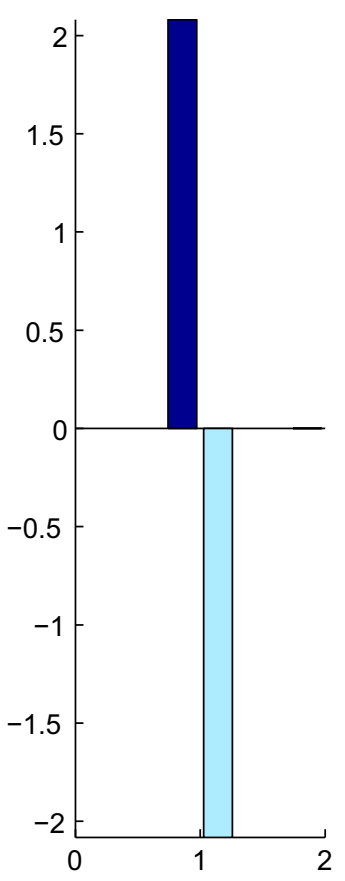

Harmonic number

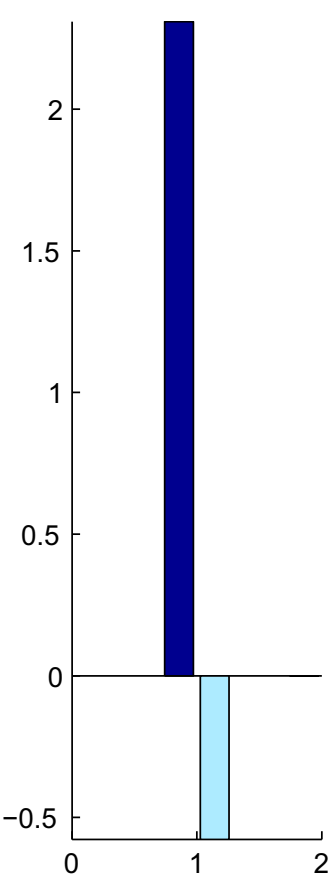

Harmonic number

Fig. 4. Solutions of Eq. (38) for $H=1$ harmonic (from left to right: mode 1, mode 2 , localized mode).
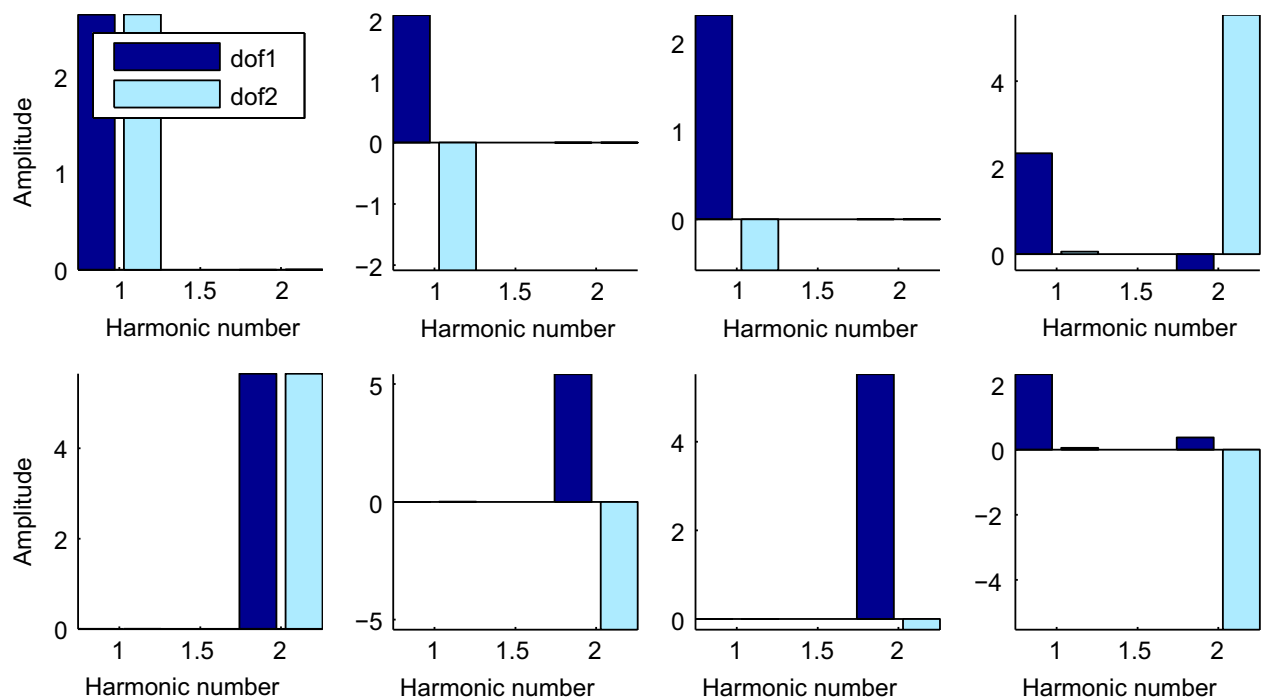

Fig. 5. Solutions of Eq. (38) for $H=2$ harmonic (from top left to bottom right: mode 1, mode 2, localized mode, internal resonance $1: 2$, subharmonic 2 mode 1 , subharmonic 2 mode 2 , subharmonic 2 localized mode, internal resonance 1:2). 

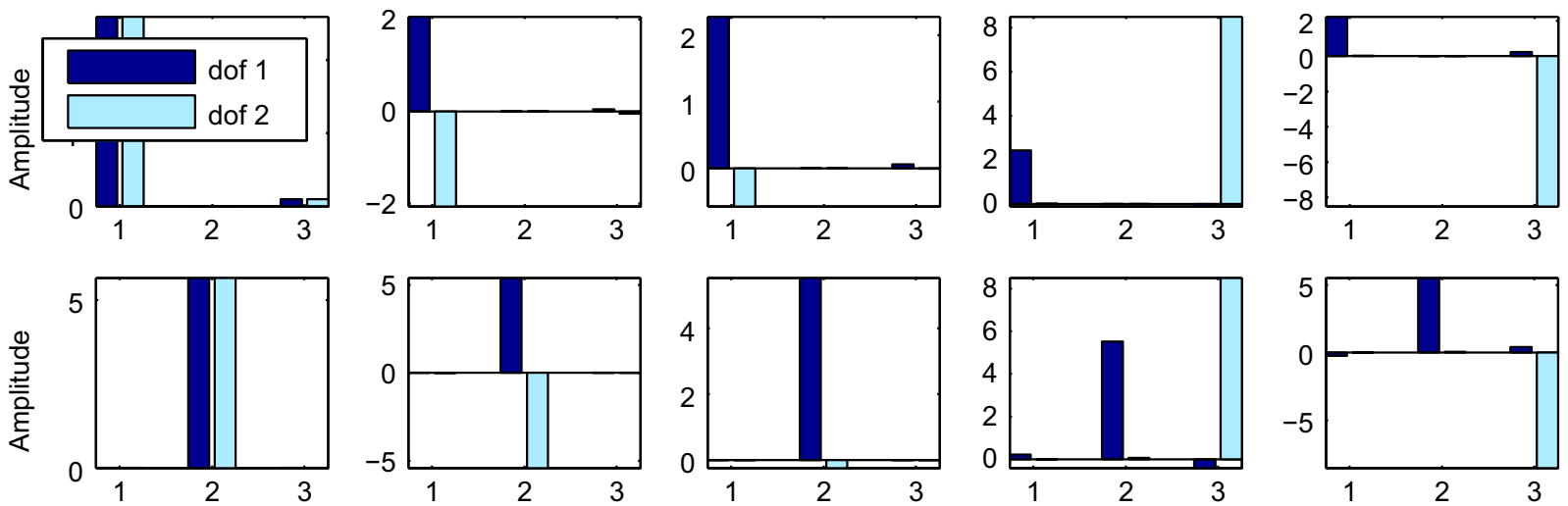

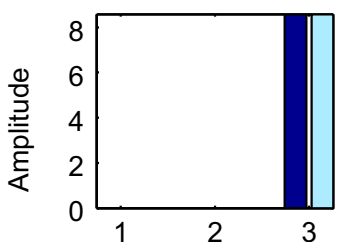

Harmonic number

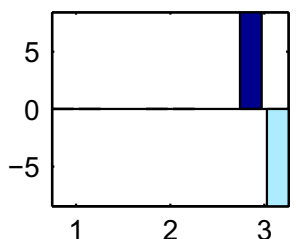

Harmonic number

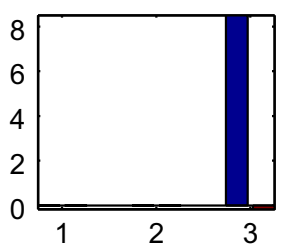

Harmonic number

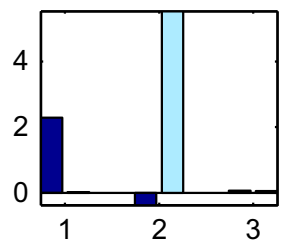

Harmonic number

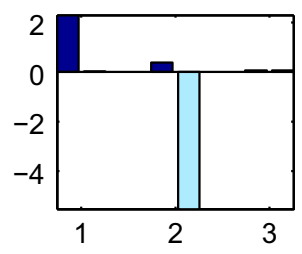

Harmonic number

Fig. 6. Solutions of Eq. (38) for $H=2$ harmonic (from top left to bottom right: mode 1, mode 2, localized mode, internal resonance $1: 3$, internal resonance 1:3, subharmonic 2 mode 1, subharmonic 2 mode 2, subharmonic 2 localized mode, internal resonance 2:3, internal resonance 2:3, subharmonic 3 mode 1 , subharmonic 3 mode 2, subharmonic 3 localized mode, internal resonance 1:2, internal resonance 1:2).

\subsection{Cyclic system}

In this section, we will apply our method for finding multiple steady states of a dynamic equation to a simple $n$ dof cyclic system, as depicted in Fig. 9. This kind of system arises, for example, when modeling a bladed disk with blades subjected to geometric nonlinearities [3]. The motion equation for such a system is defined as follows:

$$
m \ddot{u}_{i}+c \dot{u}_{i}+\left(k+2 k_{c}\right) u_{i}-k_{c} u_{i-1}-k_{c} u_{i+1}+k_{n l} u_{i}^{3}=F_{i}(t)
$$

for $i=1 \ldots n$ with convention $\boldsymbol{u}_{n+1}=\boldsymbol{u}_{1}$. Parameters $m, c, k$ are respectively the mass, damping and stiffness coefficients, $k_{c}$ and $k_{n l}$ are the coupling stiffness and nonlinear stiffness coefficients respectively and finally $F_{i}(t)$ is the excitation force, which is assumed to be in the following form: $F_{i}(t)=f \cos (\omega t)$, i.e. all dofs are forced with the same force amplitude $f$.

In the first part of the application, $n$ will be considered as variable and several elements of information on undamped solutions will be given. The particular case of $n=8$ will then be considered, and the damped solutions will be computed and displayed.

The numerical values used throughout this example are as follows:

$$
m=1, c=0.1, k=1, k_{c}=1, k_{n l}=1, f=-1 .
$$

\subsubsection{General information on the undamped system}

4.2.1.1. Linear analysis. The resonance frequency $\omega_{i}$ and mode shape $\boldsymbol{\Phi}_{i}$ of the linearized undamped system are obtained by solving the eigenvalue problem $\mathbf{K} \boldsymbol{\Phi}_{i}=\omega_{i}^{2} \mathbf{M} \boldsymbol{\Phi}_{i}$. Due to the special structure of matrix K, only one ( $n$ odd) [resp. two ( $n$ even)] single eigenvalue and $n-1$ [resp. $n-2$ ] double eigenvalues are associated with distinct eigenvectors. The results of this eigenvalue problem can be expressed as follows:

$$
\begin{array}{ll}
\omega_{1}^{2}=\frac{k}{m}, & \boldsymbol{\Phi}_{1}=[1, \ldots, 1]^{T} \\
\omega_{i}^{2}=\frac{k+2 k_{c}\left(1-\cos \left(\theta_{i}\right)\right)}{m}, & \boldsymbol{\Phi}_{i}^{(c)}=\left[\cos \left(\theta_{i}\right), \ldots, \cos \left(n \theta_{i}\right)\right]^{T}, \quad \theta_{i}=\frac{2(i-1) \pi}{n}, i \in[2, n] \\
& \boldsymbol{\Phi}_{i}^{(s)}=\left[\sin \left(\theta_{i}\right), \ldots, \sin \left(n \theta_{i}\right)\right]^{T} \\
\omega_{n}^{2}=\frac{k+2 k_{c}\left(1-\cos \left(\theta_{i}\right)\right)}{m}, & \boldsymbol{\Phi}_{i}^{(c)}=\left[\cos \left(\theta_{i}\right), \ldots, \cos \left(n \theta_{i}\right)\right]^{T}, \quad \theta_{i}=\frac{2(n-1) \pi}{n}
\end{array}
$$



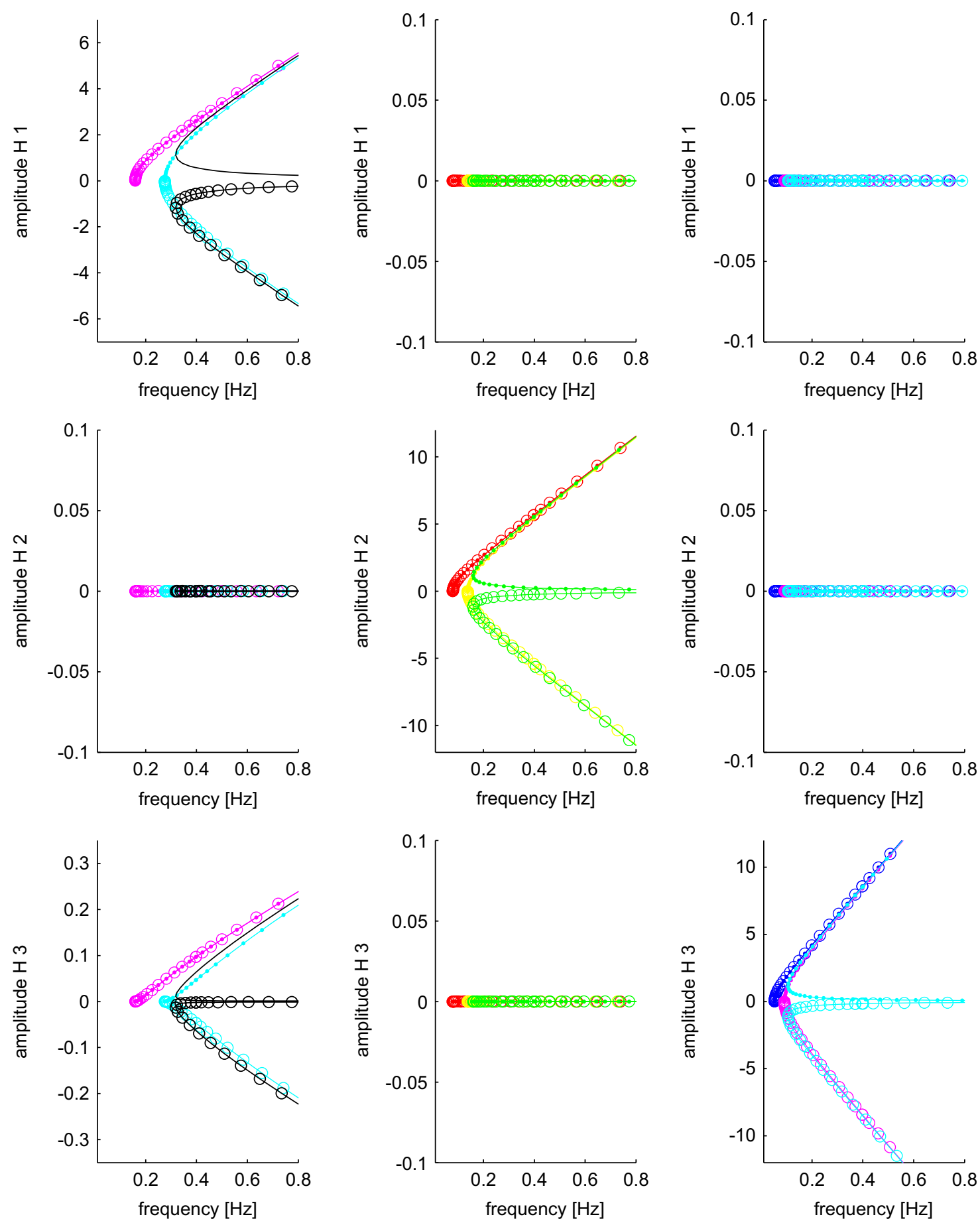

Fig. 7. Results of the frequency continuation for each NNM-like solutions: nonlinear normal modes (left), subharmonics of order 2 (middle) and subharmonics of order 3 (right) (. dof $1, \circ$ dof 2 ).

As observed above, the linear frequencies are clustered within the interval

$$
\left[\frac{1}{2 \pi} \sqrt{\frac{k}{m}}, \frac{1}{2 \pi} \sqrt{\frac{k+4 k_{c}}{m}}\right]
$$



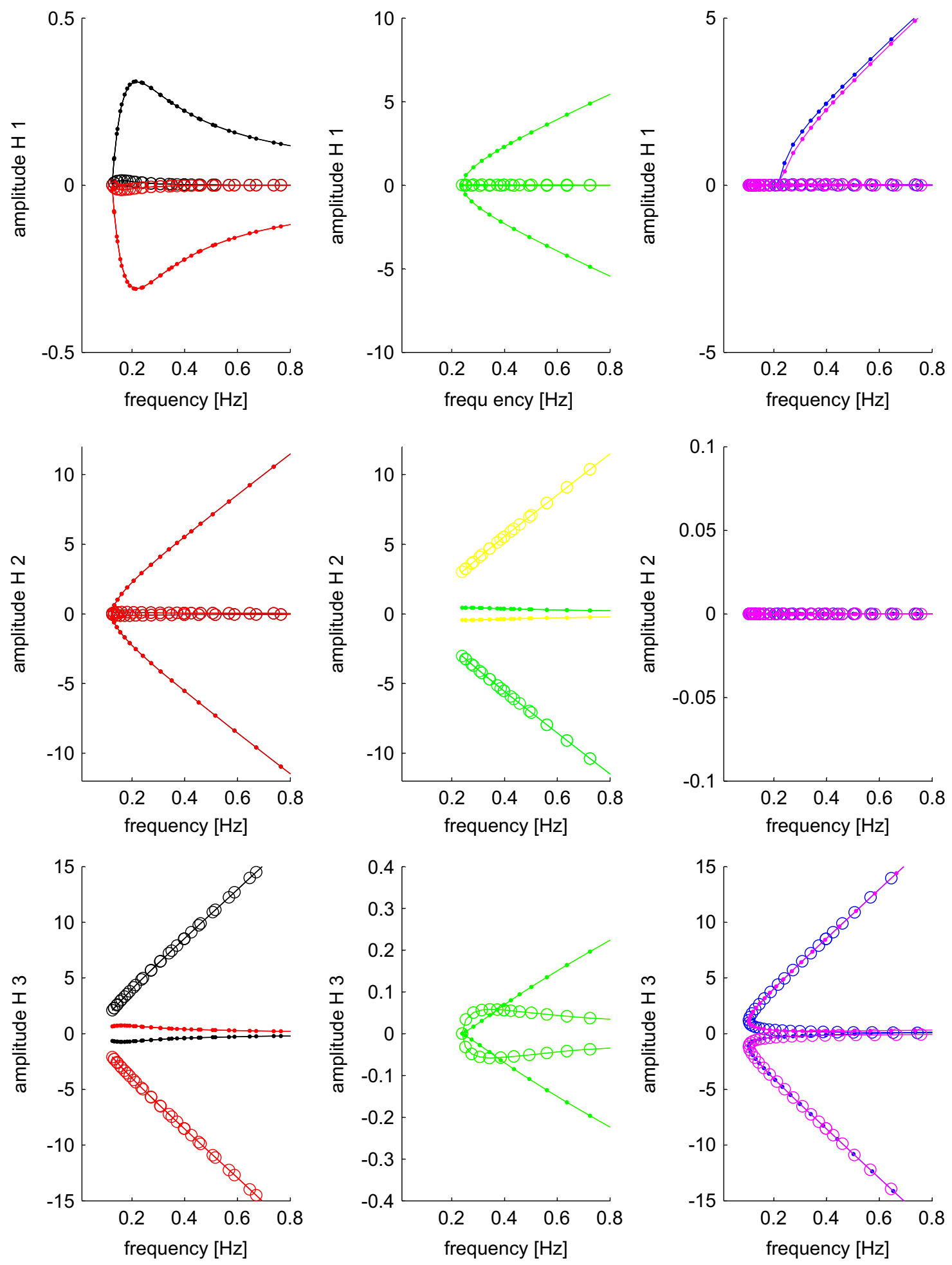

Fig. 8. Results of the frequency continuation for each internal resonance: $2: 3$ (left), 1:2 (middle), 1:3 (right) (. dof $1, \circ$ dof 2 ). 


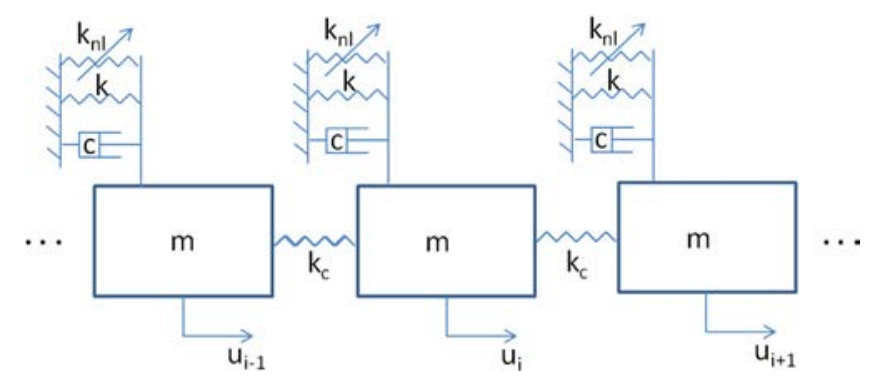

Fig. 9. Simple nonlinear cyclic system used in our example.

which is a special characteristic of cyclic systems. In the remainder of this paper, we will study this system around the first resonance frequency $\omega_{1}=\sqrt{k / m}=1$, and moreover the frequency used to search for the multiple solution will be set to $\omega_{m}=3$.

4.2.1.2. Computing multiple solutions of the undamped system. Applying the HBM with $H$ harmonics leads to an equation of the form of Eq. (4). For the computation of multiple solutions, a single harmonic approximation will be used $(H=1)$ (taking only one harmonic is especially convenient for keeping the number of variables as low as possible; this method however may be applied with any number of harmonics $H$ ).

Applying the HBM with one harmonic to the undamped system results in the following polynomial system with $n$ variables $\boldsymbol{a}=\left(a_{1}, \ldots, a_{n}\right)$ for $i=1 \ldots n$ :

$$
\left(\left(k+2 k_{c}\right)-\omega^{2} m\right) a_{i}-k_{c} a_{i-1}-k_{c} a_{i+1}+\frac{3}{4} k_{n l} a_{i}^{3}-f_{i}=p_{i}^{(u)}(\boldsymbol{a})=0
$$

As a result, the polynomial equation system $\boldsymbol{P}^{(u)}=\left(p_{1}^{(u)}, \ldots, p_{n}^{(u)}\right)$ is already in a Groebner basis form for any total degree monomial ordering. The leading term of $p_{i}^{(u)}$ is indeed $x_{i}^{3}$ (relative to any total degree ordering), thus implying that the leading terms of $p_{i}^{(u)}$ and $p_{j}^{(u)}$ are relatively prime for $i \neq j$, which results in the fact that $\boldsymbol{P}$ is a Groebner basis (see [11]). Moreover, the quotient space $\mathbb{Q}=\mathbb{C}[\boldsymbol{x}] /\left\langle\boldsymbol{P}^{(u)}\right\rangle$ is of finite dimension $D=3^{n}$.

As a consequence of the special form of polynomial system $\boldsymbol{P}^{(u)}$, no Groebner basis computation is required, and the normal set is given by $\mathcal{B}=\left\{\boldsymbol{x}^{\boldsymbol{\alpha}}, \boldsymbol{\alpha} \in[0,2]^{n}\right\}$ [29].

Solving the eigenvalue problem for multiplication matrix $\mathbf{M}_{x_{1}}$ leads to complex and real eigenvalues. In our case, we are solely interested in the real solution of Eq. (42), so only real eigenvalues have been retained. Due to the large multiplication matrix size as $n$ increases, the computation of all eigenvalues becomes very costly, and tools such as Arnoldi methods [30] may be used to extract eigenvalues with the smallest imaginary part. As matrices are filled with floating point numbers, rounding errors occur during the conversion of large rational numbers to floating point numbers, thus leading to small errors in the eigenvalue and eigenvector computations. In order to remove these errors, solutions are refined by applying a Newton-Raphson algorithm to the undamped system using the computed eigenvector as an initial guess. If the algorithm converges, the solution is retained; otherwise, we assume that the eigenvector was not a solution of our problem.

In order to take into account cyclic symmetry, we have chosen to sort our solutions by family. For a fixed frequency, a solution $\boldsymbol{a}$ is part of family $F_{\boldsymbol{a}^{*}}$ either if $\boldsymbol{a}=\boldsymbol{a}^{*}$ or if $\boldsymbol{a}$ is a circular permutation of $\boldsymbol{a}^{*}$.

Let us note that the special form of the example in the undamped case with $H=1$ always leads to a total number of solutions of $D=3^{n}$; therefore, the linear total degree homotopy method may also be efficient in this case since no divergent path will need to be followed (all $D=3^{n}$ paths lead to a solution, whether real or complex, of the undamped system) and since the homotopy method can easily be run on a parallel computer. For generic cases however, the number of solutions may be less than the Bezout bound, in which case the total degree homotopy would have to follow divergent paths. This drawback is overcome by using the Groebner basis and multiplication matrices, since they are able to provide the correct number of solutions (as the dimension of basis $\mathcal{B}$ ) and then compute them by means of eigenvalue estimation.

\subsubsection{Special case $n=8$}

The remainder of this paper will consider a system with $n=8$ dofs, where all dofs are forced with the same amplitude. While considering only 8 dofs may appear to be very simplistic, it will nonetheless be shown that this system can exhibit a wide number of steady-state solutions.

4.2.2.1. Nonlinear resonance. The first computed solution is the principal nonlinear resonance curve computed by means of the continuation method with an initial guess computed from the linear system at $\omega_{0}=0.1$. This initial solution (see Fig. 10, solution 1) corresponds to the principal resonance curve and is associated with a motion on the first linear mode shape $\boldsymbol{\Phi}_{1}$.

4.2.2.2. From undamped to damped. Next, solutions of the undamped forced system are computed using the method described in Section 3.2 for a particular frequency $\omega_{m}$ arbitrarily set to $\omega_{m}=3$. The resulting multiplication matrices are of 
size $3^{8}=6561$, thus solving the eigenvalue problem; keeping only the real solutions and sorting results by family ultimately yields a total of $N_{u}=692$ distinct solutions for the undamped forced system at $\omega_{m}=3$.

These solutions are now used as the initial iterates for continuation on the damping parameter; during the continuation procedure as damping increases, some solutions tend to disappear due to excessive damping, as explained in Section 2.3. Here, the damping continuation leads to $N_{d}=124$ solutions for the damped forced system (thus $N_{u}-N_{d}=568$ solutions have disappeared due to increased damping). The fact that the undamped solution does not always lead to a damped solution is illustrated in Fig. 2, where the solution curve can be observed to shrink as damping increases.

As a byproduct of this damping continuation method, we derive the maximum damping value for each solution to exist (i.e. the maximum value of parameter $\epsilon$ that has given a solution for the forced damped system). This information can be useful, for example, in design strategies. Another advantage of working on the undamped system is that the solutions are unbounded; hence when searching solutions at a particular frequency $\omega_{m}$, the chance of finding solutions is higher than using the damped system.

4.2.2.3. Multiple steady states of the damped system. Finally, the $N_{d}=124$ solutions of the damped system are continued using the arc-length continuation presented in Section 2.2. In order to avoid a useless continuation, we have sequentially verified that the initial point of the continuation does not belong to any previously computed solution family. This has been carried out by monitoring the distance between the initial point and all previously computed solution curves: if this distance lies below a low threshold, then the initial point is considered to be part of a previously computed solution and thus no continuation is applied. Since the system is cyclic, we have also tested to determine if the cyclic permutation of the initial point does not belong to any known solution. A similar strategy has been employed to terminate the continuation of closed curves (if the current point is near the initial point, then continuation stops).

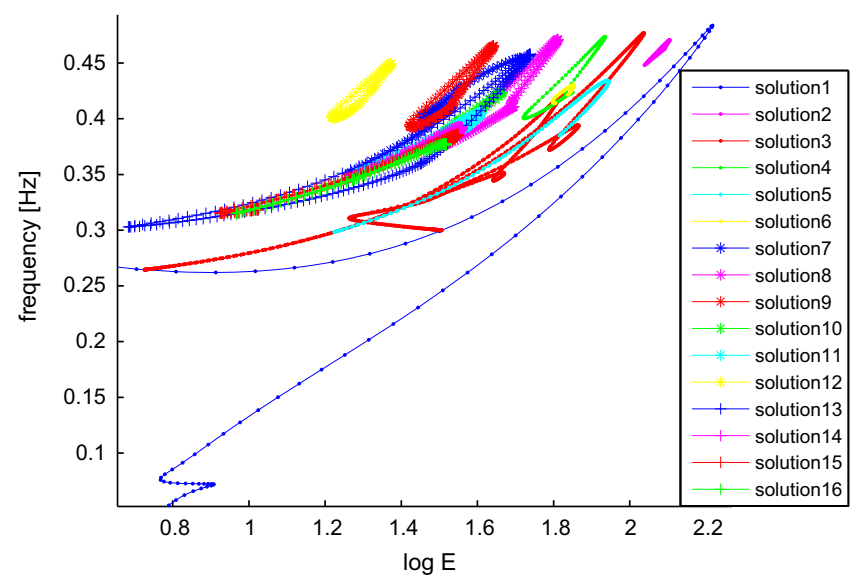

Fig. 10. Representation of all computed solutions in the energy-frequency plot.

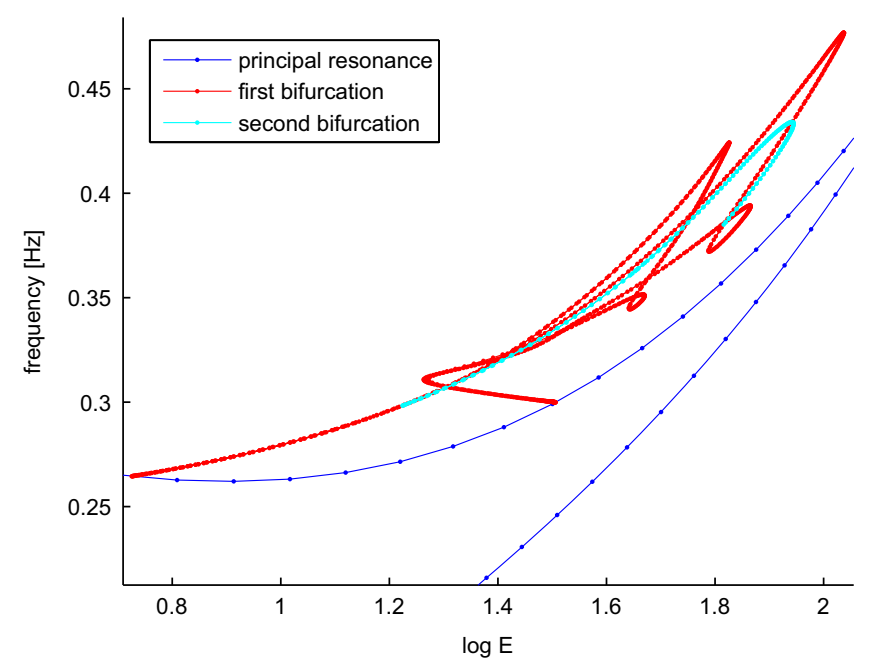

Fig. 11. Principal resonance and its bifurcation. 
Using this sequential sorting technique leads to computing only 16 continuations, corresponding to 16 families of solutions. In order to simplify the solution representation, we have opted to display results in an energy-frequency plot. A global representation of all solutions discovered is presented in Fig. 10. From this plot, it can be observed that the solutions in this example can be sorted into two categories: closed curve solutions (8), and bifurcated solutions (8). Bifurcations can arise either from the principal resonances (see Fig. 11) or from the closed curve solution (Fig. 12). In this example, some of the bifurcated solutions may be computed by monitoring the bifurcation as previously indicated in this paper (e.g. bifurcations of the principal resonance and its sub-bifurcations). Closed curve solutions however cannot be computed by this technique since they are disconnected from other solutions. The proposed method overcomes this drawback by conducting a global analysis in which the bifurcated and closed curve solutions are treated equally.

In order to complete the energy-frequency plot, the motion shapes of all solutions at maximum energy are depicted in Fig. 13. As can be observed, most of these shapes possess symmetry, except for solutions 5, 10, 11 and 14 .

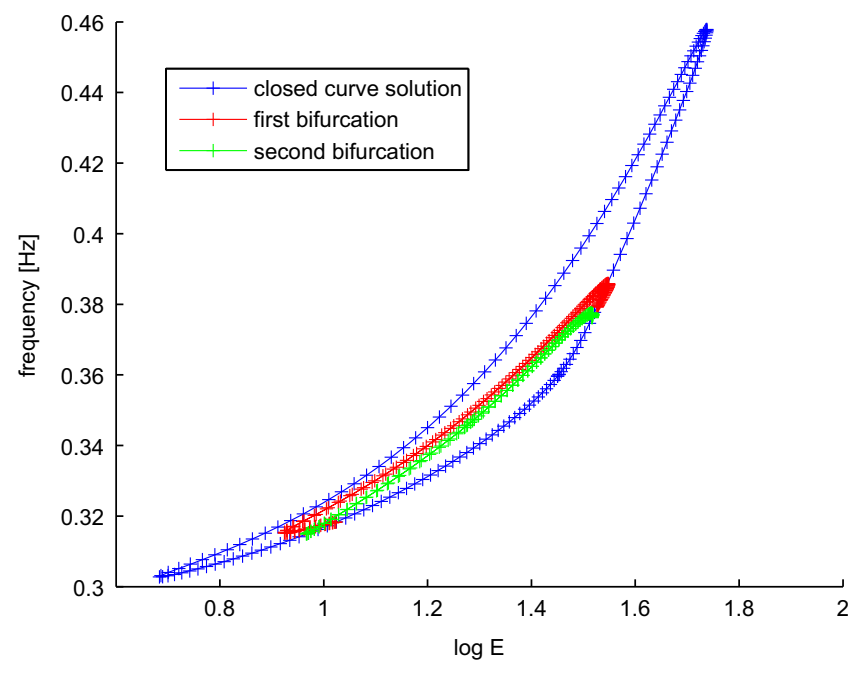

Fig. 12. A closed curve solution and its bifurcation.
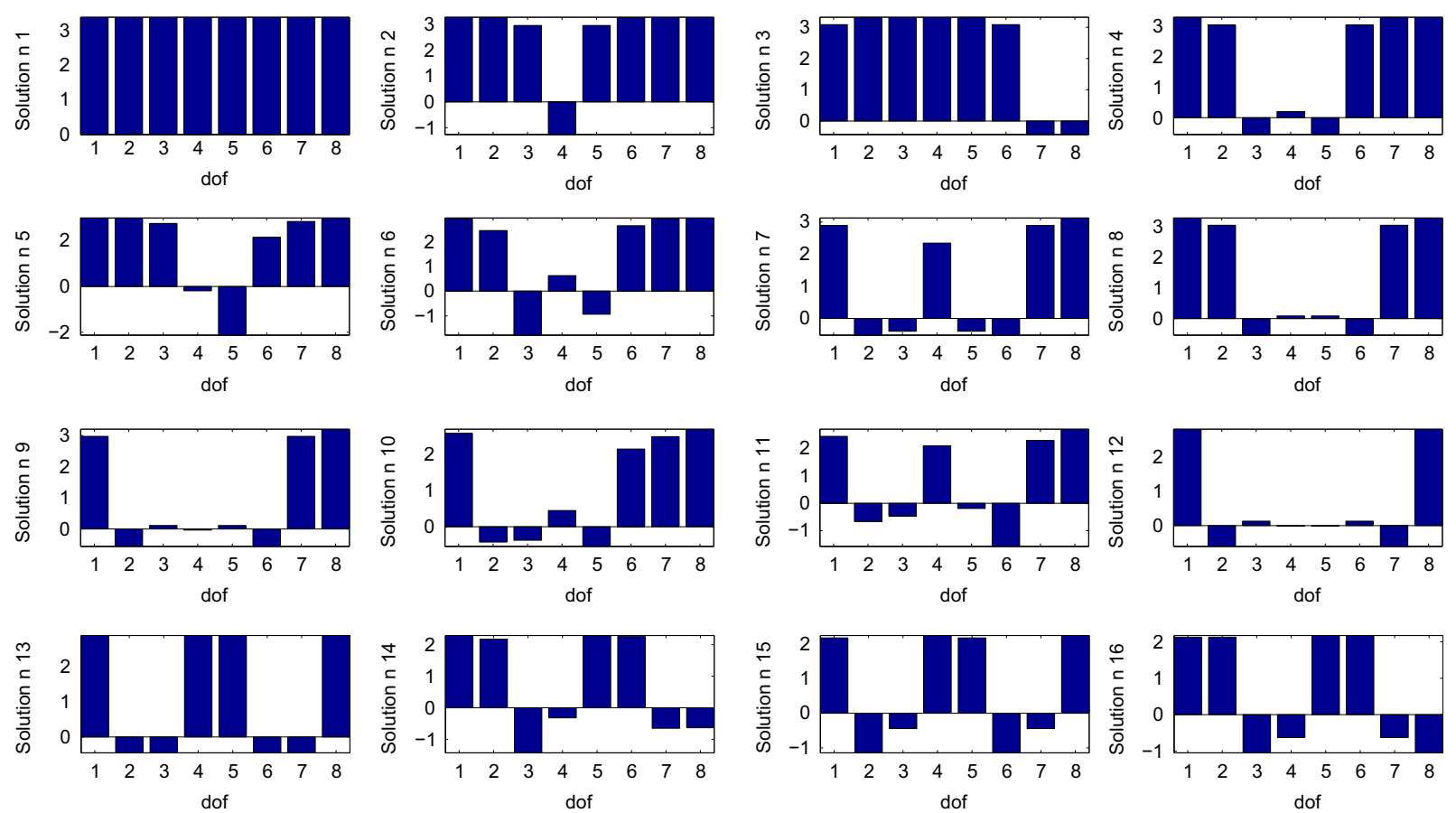

Fig. 13. Motion shape for all 16 solutions at maximum energy. 


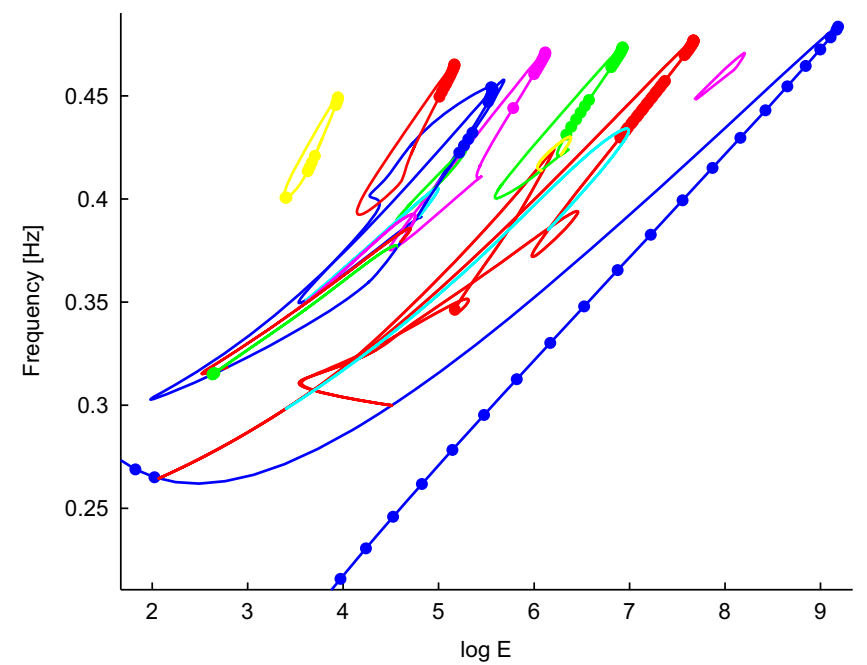

Fig. 14. Results of the stability analysis for all solutions ( $\circ$ : stable points).

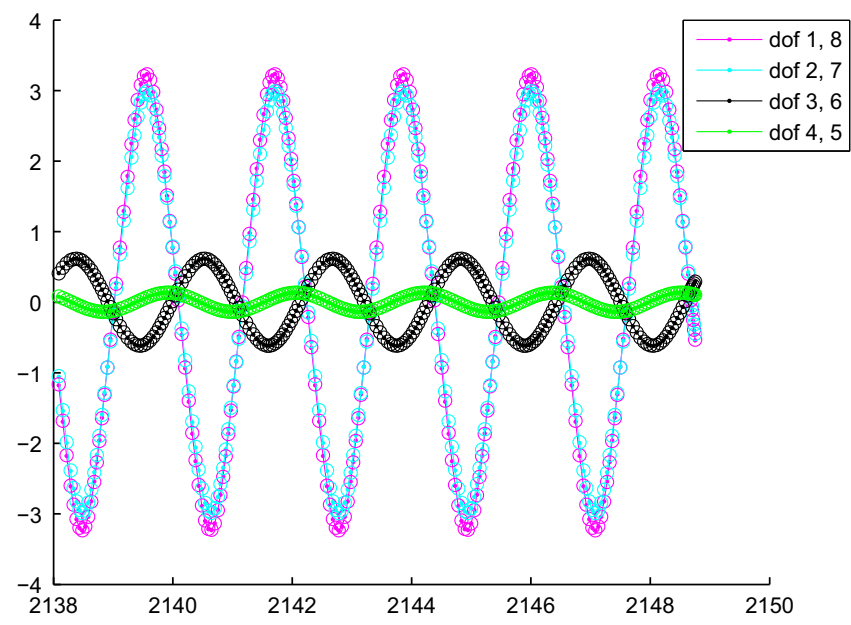

Fig. 15. Comparison between HBM (.) and temporal integration ( ${ }^{\circ}$ ), for a stable point of solution 8 at $f=0.46539 \mathrm{~Hz}$.

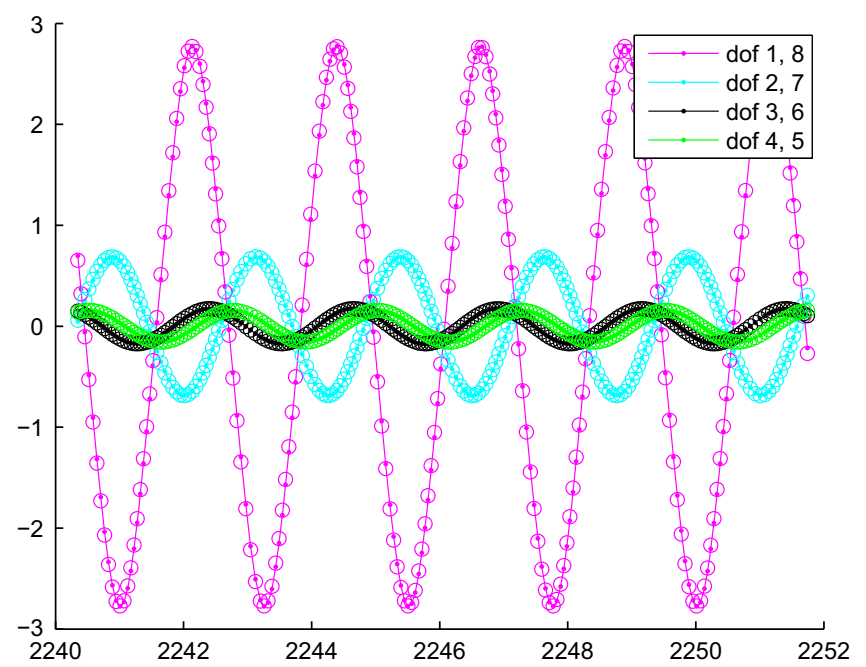

Fig. 16. Comparison between HBM (.) and temporal integration ( ${ }^{\circ}$, for a stable point of solution 12 at $f=0.4441 \mathrm{~Hz}$. 


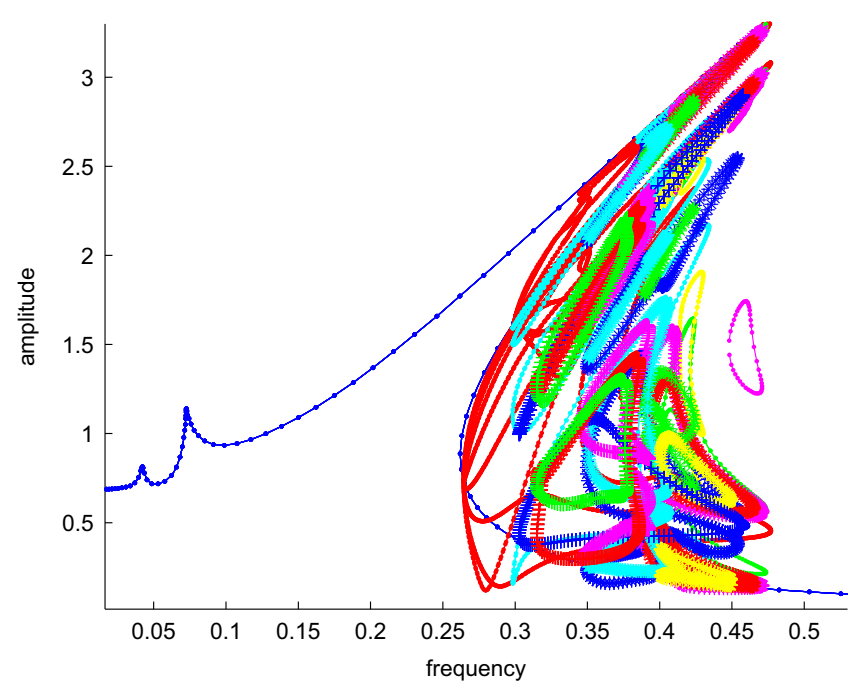

Fig. 17. Representation of all solutions of the damped system in the frequency-amplitude plot (16 solution families have been plotted).

In addition, the solution stability was computed using Floquet's theory and monodromy matrices. Results are shown in Fig. 14. Let us note that only a small number of points are stable, but almost every solution is stable near its maximum energy.

In order to validate the HBM solutions and stability analysis, temporal integrations were performed on several stable solution points. This integration procedure is carried out over 1000 periods and only the 5 last periods are retained. Comparisons of temporal integrations and HBM results are displayed in Figs. 15 and 16; a very good agreement is shown between HBM and temporal integration solutions.

Moreover, all solutions are depicted in a classical frequency-amplitude plot in order to showcase the level of complexity of system dynamics (see Fig. 17).

\section{Conclusion}

This paper has proposed a method to compute multiple steady states of nonlinear dynamic equations with polynomial nonlinearities. The harmonic balance method has been used to transform the set of nonlinear differential equations into polynomial equations, which can then be solved by computation in the induced quotient algebra. In order to decrease computation time, we have proposed working on the undamped system first. This procedure offers several advantages, among which (i) a decrease in the number of variables, and (ii) placement of the polynomial system in Groebner basis form so that no Groebner basis computations are needed. Once the undamped system has been totally solved, the solutions of the damped system are recovered by the use of a continuation method on the damping parameter, thus leading to a full frequency response diagram.

The proposed method has been applied to a simple example with 8 dofs. The overall procedure is motivated by the fact that nonlinear normal modes forms backbones curves for the forced solutions. Ideally, we should start with nonlinear normal modes, then find forced undamped solutions, and finally apply the damping continuation procedure. Here we choose to start from the undamped forced solutions as they are structurally close to the forced and damped solutions. Using continuity arguments, we suppose that all solutions of the forced and damped systems tend to the solution of the undamped forced system as the damping goes to zero. Taking the argument in the reverse way, we can suppose that all solutions of the damped forced system can be obtained from a suitable solution of the undamped forced system using continuation procedure. Of course, if we only consider a subset of solution of the undamped forced system, the continuation procedure on the damping parameter will only lead to a subset of solution of the damped and forced system. In this paper we only consider monophase solution of the undamped forced system, leading to monophase solution of the damped and forced system (solution where all dofs are "in-phase" with the excitation). However, the undamped forced system can have other types of solution such as travelling waves, or internal resonances that cannot be obtained with the "monophase hypothesis". In this case, one has to compute those additional solution before applying the damping continuation procedure. Futur work is planned to elaborate further on this point.

In this paper, the solutions of polynomial systems are computed by solving eigenvalue problems related to multiplication matrices. The major limitation of this method is clearly the exponential growth in multiplication matrix size with the number of variables. For a system with cubic nonlinearity, the maximum number of allowable degrees of freedom to have relatively short computation times seems to lie around 10 (i.e. multiplication matrices of size $3^{10} \approx 6 \times 10^{4}$ ). In being careful about memory management, using sparse multiplication matrices along with a parallel eigenvalue solver may increase this limit. It is relatively certain however that a system with more than 30 dofs (i.e. matrix size $\approx 10^{14}$ ) cannot be fully solved 
with this method within a reasonable amount of time. In addition, since the system has cyclic symmetry, a high number of redundant solutions (up to a circular shift of their component) is obtained. We have chosen herein to handle the redundant solution in a heuristic way by sorting the solutions by family. Nonetheless, it may be useful to consider symmetry before solving the system in order to reduce the number of solutions. Finally, to maintain the number of variables sufficiently small, model order reduction methods adapted to nonlinear problems should also be considered.

\section{References}

[1] G. Kerschen, M. Peeters, J.C. Golinval, A.F. Vakakis, Nonlinear normal modes. Part I. A useful framework for the structural dynamicist, Mech. Syst. Signal Process. 23 (2009) 170-194.

[2] A.F. Vakakis, Dynamics of a nonlinear periodic structure with cyclic symmetry, Acta Mech. 95 (1992) $197-226$.

[3] E. Sarrouy, A. Grolet, F. Thouverez, Global and bifurcation analysis of a structure with cyclic symmetry, Int. J. Nonlinear Mech. 46 (2011) $727-737$.

[4] L. Salles, L. Blanc, F. Thouverez, A.M. Gouskov, Dynamic analysis of fretting-wear in friction contact interfaces, Int. J. Solid Struct. 2011 (2011) 1513-1524.

[5] G. Groll, D.J. Ewins, The harmonic balance method with arc-length continuation in rotor stator contact problems, J. Sound Vib. 241 (2) (2001) 223-233.

[6] A.H. Nayfey, B. Balanchandran, Applied nonlinear dynamics: analytical, computational and experimental methods, John Wiley \& Sons, 2008.

[7] C.S. Liu, W. Yeih, Atluri Satya, An enhanced fictuous time integration method for nonlinear algebraic equation with multiple solutions, Comput. Model, Eng. Sci. 59 (3) (2010) 301-323.

[8] T.Y. Li, W. Wang, The bkk root count in $c^{n}$, Math. Comput. 65 (216) (1996) 1477-1484.

[9] T. Gao, T.Y. Li, X. Wang, Finding all isolated zeros of polynomial systems in $c^{n}$ via stables mixed volumes, J. Symb. Comput. 28 (1999) 187-211.

[10] T.Y. Li, Solving polynomial systems with polyhedral homotopie, Taiwan. J. Math. 3 (1999) 251-279.

[11] D.A. Cox, J. Little, D. O'Shea, Ideals, Varieties, and Algorithms: An Introduction to Computational Algebraic Geometry and Commutative Algebra, Undergraduate Texts in Mathematics, Springer, New York, 2010, ISBN: 9781441922571, http://books.google.fr/books?id=KxV2cgAACAAJ.

[12] F. Rouillier, Solving zero dimensional polynomial system through the rational univariate representation, Appl. Algebra Eng., Commun. Comput. 9 (1999) 433-461.

[13] J. Keyser, K. Ouchi, J.M. Rojas, The exact rational univariate representation and its application. AMS/DIMACS Volume on Computer Aided Design and Manufacturing, American Mathematical Society/Center for Discrete Mathematics and Computer Science, 2005.

[14] W. Auzinger, H.J. Stetter, An elimination algorithm for the computation of all zeros of a system of multivariate polynomial equations.

[15] H.M. Moller, R. Tenberg, Multivariate polynomial system solving using intersections of eigenspaces, J. Symb. Comput. 32 (2001) 513-531.

[16] H.M. Moller, H.J. Stetter, Multivariate polynomial equations with multiple zeros solved by matrix eigenproblems, Numer. Math. 70 (1995) 311-329.

[17] R.W. VanDervort, Geometrically nonlinear analysis of rectangular orthotropic plates using Groebner basis (Ph.D. thesis), Tennessee Technological University, 2009.

[18] A. Shanmugasundaram, An application of the method of Groebner bases to a geometrically nonlinear free vibration analysis of composite plates (Ph.D. thesis), Tennessee Technological University, 2009.

[19] T.M. Cameron, J.H. Griffin, P. Grant, An alternating frequency time domain method for calculating the steady state response of nonlinear dynamic systems, J. Appl. Mech. 56 (1) (1989) 149-154.

[20] E. Sarrouy, Analyse globale de systèmes mécaniques non linéaires. Application a la dynamique des rotors (Ph.D. thesis), Ecole Centrale Lyon, 2008.

[21] C.S. Hsu, Cell to Cell Mapping-A Method of Global Analysis for Nonlinear Systems, Applied Mathematical Sciences 64 (1987).

[22] B. Buchberger, An algorithm for finding the basis element of residue class ring of a zero dimensional polynomial ideal (Ph.D. thesis), J. Kepler University, 1965.

[23] J.C. Faugere, P. Gianni, D. Mora, Efficient computation of zero-dimensional Groebner basis by change of ordering, J. Symb. Comput. 16 (1993) 329-344.

[24] J.C. Faugere, A new efficient algorithm for computing Groebner basis (f4), 2002.

[25] J.C. Faugere, A new efficient algorithm for computing Groebner basis without reduction to zero (f5), 2004.

[26] B. Buchberger, Groebner Basis, A Short Introduction for Systems Theorists.

[27] D. Lazard, Thirty years of polynomial system solving, and now? J. Symb. Comput. 44 (2009) $222-231$.

[28] A.J. Sommese, C.W. Wampler, The Numerical Solution of Systems of Polynomials Arising in Engineering and Science, World Scientific, 2005, ISBN: 9789812567727, http://books.google.fr/books?id=S6flrWaFNOsC.

[29] B. Hanzon, D. Jibetean, Global minimization of a multivariate polynomial using matrix methods, J. Glob. Optim. 27 (2003) 1-23.

[30] R.B. Lehoucq, D.C. Sorensen, C. Yang, ARPACK Users' Guide: Solution of Large-scale Eigenvalue Problems with Implicitly Restarted Arnoldi Methods, Software, environments, tools, SIAM, 1998, ISBN: 9780898714074, http://books.google.fr/books?id=d3YnKxJpILcC. 